\title{
Uniqueness of Entropy Solutions of Nonlinear Elliptic-Parabolic-Hyperbolic Problems in One Dimension Space
}

\author{
Stanislas OUARO \\ Laboratoire d'Analyse Mathématique des Equations (LAME) \\ UFR. Sciences Exactes et Appliquées \\ Université de Ouagadougou \\ 03 BP 7021 Ouaga 03 \\ Ouagadougou - Burkina Faso \\ souaro@univ-ouaga.bf
}

Received: November 20, 2006

Accepted: November 19, 2007

\begin{abstract}
We consider a class of elliptic-parabolic-hyperbolic degenerate equations of the form $b(u)_{t}-a\left(u, \varphi(u)_{x}\right)_{x}=f$ with homogeneous Dirichlet conditions and initial conditions. In this paper we prove an $L^{1}$-contraction principle and the uniqueness of entropy solutions under rather general assumptions on the data.
\end{abstract}

Key words: elliptic, parabolic, hyperbolic, weak solution, entropy solution, $L^{1}$-contraction principle.

2000 Mathematics Subject Classification: 35K65, 35L65.

\section{Introduction}

Let $I$ be an open bounded interval of $\mathbb{R}$. We consider the initial-boundary-value problem

$$
\begin{cases}b(u)_{t}-a\left(u, \varphi(u)_{x}\right)_{x}=f & \text { in } \mathbb{Q}=] 0, T[\times I, \\ b(u)=v_{0} & \text { on }\{0\} \times I, \\ u=0 & \text { on } \Gamma=] 0, T[\times \partial I,\end{cases}
$$

Suported by ICTP under SIDA. 
with $T>0$, where

$a:(z, \xi) \in \mathbb{R} \times \mathbb{R} \longrightarrow \mathbb{R}$ is continuous, nondecreasing in $\xi \in \mathbb{R}$ with $a(0,0)=0$

$b: \mathbb{R} \longrightarrow \mathbb{R}$ is continuous, nondecreasing and surjective with $b(0)=0$;

$\varphi: \mathbb{R} \longrightarrow \mathbb{R}$ is continuous, nondecreasing with $\varphi(0)=0$.

Whenever $u$ is such that $b(u)$ is constant, (EP) degenerates into an elliptic problem of the form

$$
\begin{cases}-a\left(u, \varphi(u)_{x}\right)_{x}=f & \text { in } \mathbb{Q}=] 0, T[\times I, \\ b(u)=v_{0} & \text { on }\{0\} \times I, \\ u=0 & \text { on } \Gamma=] 0, T[\times \partial I .\end{cases}
$$

If we let $b=\mathrm{id}$, on each part where $u$ is such that $\varphi(u)$ is constant, then (EP) degenerates to a scalar conservation law of the form

$$
\begin{cases}u_{t}-a(u, 0)_{x}=f & \text { in } \mathbb{Q}=] 0, T[\times I, \\ u=u_{0} & \text { on }\{0\} \times I, \\ u=0 & \text { on } \Gamma=] 0, T[\times \partial I .\end{cases}
$$

It is then clear that we include in (EP), some first order hyperbolic problems, for which, even under assumptions of regularity on data, there is no hope to get classical global solutions.

It is also well known that, for such equations, the above problems are ill-posed in the sense that there is no uniqueness. It is therefore necessary to introduce Kruzhkov solutions in order to obtain existence and uniqueness results (see [16]).

Since $b$ and $\varphi$ are not strictly increasing, the formulations considered above include Stefan problems, filtration problems, etc., in the one dimensional case. Such formulations involve a large class of problems and an important literature has been developed. The case $b=\mathrm{id}$ for the problems that we consider in this paper was studied by Bénilan and Touré [8], where they proved existence of entropy solutions under assumptions of the generalized domain. (Uniqueness of entropy solution remained an open problem under such condition only). They also proved (with an additional condition on the data such as $\varphi^{-1} \in C(\mathbb{R})$ ) existence and uniqueness of entropy solution of the problem considered here. Note also that, in a bounded domain of $\mathbb{R}^{N}$, when $\varphi=\mathrm{id}$, under additional assumption on the vector field $a$, Carrillo and Wittbold (see [12]) have proved uniqueness and a comparison result for weak solutions and, more generally, renormalized solutions of the problem

$$
\begin{cases}b(u)_{t}-\operatorname{div} a(u, D u)=f & \text { in } \mathbb{Q}=] 0, T[\times \Omega, \\ b(u)=v_{0} & \text { on }\{0\} \times \Omega, \\ u=0 & \text { on } \Gamma=] 0, T[\times \partial \Omega .\end{cases}
$$

In this paper we prove uniqueness and a comparison result for entropy solution of (EP). Existence of entropy solutions was proved by the author (see [19]). We 
will briefly recall the results of [19] in section 1, and section 2 is devoted to the main theorem of this paper.

\section{Preliminaries}

Let $a, b$, and $\varphi$ be given functions such that

$$
a: \mathbb{R} \times \mathbb{R} \longrightarrow \mathbb{R}, \quad \varphi: \mathbb{R} \longrightarrow \mathbb{R}, \quad b: \mathbb{R} \longrightarrow \mathbb{R} \text { are continuous. }
$$

We make the following assumptions:

$$
a(k, \xi) \text { is nondecreasing in } \xi,
$$

$b(k)$ and $\varphi(k)$ are nondecreasing and $b$ is surjective,

$$
a(0,0)=b(0)=\varphi(0)=0 .
$$

Define

and

$$
\begin{aligned}
H(k) & =a(k, 0) \text { for } k \in \mathbb{R}, \quad h=a\left(u, \varphi(u)_{x}\right), \\
H^{+}(s) & = \begin{cases}1 & \text { if } s>0, \\
{[0,1]} & \text { if } s=0, \\
0 & \text { if } s<0,\end{cases} \\
H_{\epsilon}(s) & =\min \left(\frac{s^{+}}{\epsilon}, 1\right),
\end{aligned}
$$

$$
H_{0}(s)= \begin{cases}1 & \text { if } s>0 \\ 0 & \text { otherwise }\end{cases}
$$

Let $\gamma$ be a maximal monotone operator defined on $\mathbb{R}$. We denote by $\gamma_{0}$ the main section of $\gamma$ :

$$
\gamma_{0}(s)= \begin{cases}\text { the element of minimal absolute value of } \gamma(s) & \text { if } \gamma(s) \neq \emptyset, \\ +\infty & \text { if }[s,+\infty) \cap D(\gamma)=\emptyset, \\ -\infty & \text { if }(-\infty, s] \cap D(\gamma)=\emptyset,\end{cases}
$$

where $D(\gamma)$ is the domain of $\gamma$.

Our main assumption is the coerciveness of $a$ with respect to $\xi$, for $k$ bounded; more precisely,

$$
\lim _{|\xi| \rightarrow \infty} \inf _{|k|<R}|a(k, \xi)|=+\infty \quad \forall R>0 .
$$

We also assume the following hypotheses:

$$
\begin{aligned}
(a(r, \xi)-a(s, \eta)) \cdot(\xi-\eta)+M(r, s)(1 & \left.+|\xi|^{2}+|\eta|^{2}\right)|\varphi(r)-\varphi(s)| \\
& \geq \Gamma(\varphi(r), \varphi(s)) \cdot \xi+\hat{\Gamma}(\varphi(r), \varphi(s)) \cdot \eta
\end{aligned}
$$


for all $r, s, \xi, \eta \in \mathbb{R}$, where $M: \mathbb{R} \times \mathbb{R} \rightarrow \mathbb{R}^{+}, \Gamma, \hat{\Gamma}: \mathbb{R} \times \mathbb{R} \rightarrow \mathbb{R}$ are continuous functions;

There exists $\hat{a}: \mathbb{R} \times \mathbb{R} \rightarrow \mathbb{R}$ continuous, nondecreasing with respect to the second variable and such that $\hat{a}\left(b(u), \varphi(u)_{x}\right)=a\left(u, \varphi(u)_{x}\right)$.

$$
(a(z, \xi)-a(z, 0)) \cdot \xi \geq \lambda|\xi|^{2},
$$

where $\lambda>0$.

\section{Remarks 1.1.}

(i) Assumption $\left(\mathrm{H}_{2}\right)$ implies $\Gamma(\varphi(r), \varphi(r))=\hat{\Gamma}(\varphi(r), \varphi(r))=0$ for all $r \in \mathbb{R}$. Indeed, choosing $r=s, \eta=0, \xi=t \nu, t>0, \nu \in \mathbb{R}$ in $\left(\mathrm{H}_{2}\right)$, we get $t \nu[a(r, t \nu)-a(r, 0)] \geq \Gamma(\varphi(r), \varphi(r)) t \nu$. Dividing by $t$ and taking limit as $t \rightarrow 0$, we get $\Gamma(\varphi(r), \varphi(r)) \nu \leq 0$ for all $\nu \in \mathbb{R}$; hence $\Gamma(\varphi(r), \varphi(r))=0$. Using the same argument we obtain the corresponding result for $\hat{\Gamma}$.

(ii) $\left(\mathrm{H}_{2}\right)$ implies that $a$ is monotone with respect to the second variable (see $[12$, Remark 2.2] for the proof).

We now define the $L^{1}(I)$ operator associated with the evolution problem (EP) by $A_{b} b(u)=-a\left(u, \varphi(u)_{x}\right)_{x}$ and it satisfies

$v \in A_{b} b(u)$ if and only if $b(u) \in L^{1}(I), v \in L^{\infty}(I)$, and $u$ is an entropy solution of the stationary problem (SP) with $f=v+b(u)$,

where

$$
\begin{cases}b(u)-a\left(u, \varphi(u)_{x}\right)_{x}=f & \text { in } I, \\ u=0 & \text { on } \Gamma=\partial I .\end{cases}
$$

We have showed for this operator (see [18, Proposition 4.1] for the proof), the following result:

Lemma 1.2. Suppose that $\left(\mathrm{H}_{1}\right)$ and $\left(\mathrm{H}_{2}\right)$ are satisfied. Then the operator $A_{b}$ defined above satisfies the following:

(i) $A_{b}$ is $T$-accretive in $L^{1}(I)$, i.e.,

$$
\left\|(x-\tilde{x})^{+}\right\|_{L^{1}} \leq\left\|\left(x-\tilde{x}+\lambda\left(A_{b} x-A_{b} \tilde{x}\right)\right)^{+}\right\|_{L^{1}}
$$

for all $\lambda \geq 0$ and $x, \tilde{x} \in D\left(A_{b}\right)$.

(ii) For any $\lambda>0$, the range $R\left(I+\lambda A_{b}\right)$ of $I+\lambda A_{b}$ is dense in $L^{1}(I)$.

(iii) The domain $D\left(A_{b}\right)$ of $A_{b}$ is dense $i n L^{1}(I)$.

We now recall the definition of weak and entropy solutions of (EP). 
Definition 1.3. Let $f \in L^{2}\left((0, T) ; H^{-1}(I)\right)$ and $v_{0} \in L^{1}(I)$. A weak solution of problem (EP) is a measurable function $u$ which also satisfies the following:

$$
\begin{gathered}
b(u) \in L^{1}(Q), \quad b(u)_{t} \in L^{2}\left(0, T ; H^{-1}(I)\right), \\
\varphi(u) \in L^{2}\left(0, T ; H_{0}^{1}(I)\right), \quad h=a\left(u, \varphi(u)_{x}\right) \in L^{2}(\mathbb{Q}), \\
b(u)_{t}-h_{x}=f \quad \text { in } D^{\prime}(\mathbb{Q}), \\
b(u(0, x))=v_{0}(x) \quad \text { a.e. on } I .
\end{gathered}
$$

The last condition should be understood in the following sense:

$$
\int_{0}^{T}\left\langle b(u)_{t}, \xi\right\rangle d t=-\int_{\mathbb{Q}} b(u) \xi_{t} d x d t-\int_{I} v_{0} \xi(0) d x
$$

for any $\xi \in L^{2}\left((0, T) ; H_{0}^{1}(I)\right) \cap W^{1,1}\left((0, T) ; L^{\infty}(I)\right)$, such that $\xi(T)=0$, where $\langle\cdot, \cdot\rangle$ represents the duality pairing between $H^{-1}(I)$ and $H_{0}^{1}(I)$.

Remark 1.4. We easily check that if $u$ is a weak solution of $(\mathrm{EP})(b, a, \varphi, f)$ then $(-u)$ is a weak solution of $(\mathrm{EP})(\tilde{b}, \tilde{a}, \tilde{\varphi},-f)$ where $\tilde{b}(s)=-b(-s), \tilde{\varphi}=-\varphi(-s)$, and $\tilde{a}(s, k)=-a(-s,-k)$.

Definition 1.5. Let $f \in L^{2}\left((0, T) ; H^{-1}(I)\right) \cap L^{1}(\mathbb{Q})$ and $v_{0} \in L^{1}(I)$. An entropy solution of problem (EP) is a weak solution $u$ which satisfies the following:

$$
\begin{aligned}
\int_{\mathbb{Q}} H_{0}(u-k)\left\{\xi_{x}(h-H(k))-(b(u)-b(k)) \xi_{t}-f \xi\right\} d x d t & \\
& -\int_{I}\left(v_{0}-b(k)\right)^{+} \xi(0) d x \leq 0
\end{aligned}
$$

for any $(k, \xi) \in \mathbb{R} \times\left(L^{2}\left((0, T) ; H^{1}(I)\right) \cap W^{1,1}\left((0, T) ; L^{\infty}(I)\right)\right)$ such that $k \geq 0, \xi \geq 0$, and $\xi(T)=0$ and for any $(k, \xi) \in \mathbb{R} \times\left(L^{2}\left((0, T) ; H_{0}^{1}(I)\right) \cap W^{1,1}\left((0, T) ; L^{\infty}(I)\right)\right)$ such that $\xi \geq 0$ and $\xi(T)=0$;

$$
\begin{aligned}
\int_{\mathbb{Q}} H_{0}(k-u)\left\{\xi_{x}(h-H(k))-(b(u)-b(k)) \xi_{t}-\right. & f \xi\} d x d t \\
& +\int_{\mathbb{R}}\left(v_{0}-b(k)\right)^{-} \xi(0) d x \geq 0
\end{aligned}
$$

for any $(k, \xi) \in \mathbb{R} \times\left(L^{2}\left((0, T) ; H^{1}(I)\right) \cap W^{1,1}\left((0, T) ; L^{\infty}(I)\right)\right)$ such that $k \leq 0, \xi \geq 0$, and $\xi(T)=0$ and for any $(k, \xi) \in \mathbb{R} \times\left(L^{2}\left((0, T) ; H_{0}^{1}(I)\right) \cap W^{1,1}\left((0, T) ; L^{\infty}(I)\right)\right)$ such that $\xi \geq 0$ and $\xi(T)=0$.

Remark 1.6. It is easy to see that if $u$ is an entropy solution of $(\mathrm{EP})(b, a, \varphi, f)$ then $(-u)$ is an entropy solution of $(\mathrm{EP})(\tilde{b}, \tilde{a}, \tilde{\varphi}, \tilde{f})$ where $\tilde{b}(r)=-b(-r), \tilde{a}(r, k)=$ $-a(-r,-k), \tilde{\varphi}(r)=-\varphi(-r)$, and $\tilde{f}=-f$.

We then have the following lemma (see [19]).

Lemma 1.7. Let $\left(\mathrm{H}_{1}\right)-\left(\mathrm{H}_{4}\right)$ hold, then $(\mathrm{EP})$ has at least an entropy solution. 


\section{Comparison result and uniqueness}

We will study in this section the question of uniqueness of the entropy solution of the evolution problem (EP) which is the main result of this paper (see Theorem 2.5 below).

Remark 2.1. The concept of uniqueness considered here is the uniqueness of $b(u)$; on the other hand, if $b$ is one to one, the uniqueness of $b(u)$ is equivalent to that of $u$ (see [9] for more details).

For the proof of uniqueness, we use a method developed by Carrillo (see [11]) and Carrillo-Wittbold (see [12]) for parabolic degenerated problems. We start by showing that entropy solutions satisfy Kato's inequality (cf. [4]); more precisely we show that entropy solutions satisfy the following inequality:

Theorem 2.2 (Kato's Inequality). For $i=1,2$, let $f_{i} \in L^{2}\left((0, T) ; H^{-1}(I)\right) \cap L^{1}(\mathbb{Q})$ and $v_{0_{i}} \in L^{1}(I)$, let $u_{i}$ be an entropy solution of $(\mathrm{EP})$ with respect to data $\left(f_{i}, v_{0_{i}}\right)$. Then

$$
\begin{aligned}
\int_{\mathbb{Q}} H_{0}\left(u_{1}-u_{2}\right)\left(h_{1}-h_{2}\right) \xi_{x} d x d t-\int_{\mathbb{Q}}\left(b\left(u_{1}\right)-b\left(u_{2}\right)\right)^{+} \xi_{t} d x d t \\
-\int_{I}\left(v_{0_{1}}-v_{0_{2}}\right)^{+} \xi(0) d x \leq \int_{\mathbb{Q}} H_{0}\left(u_{1}-u_{2}\right)\left(f_{1}-f_{2}\right) \xi d x d t
\end{aligned}
$$

for any nonnegative $\xi \in D([0, T) \times I)$.

For the proof of theorem 2.2, we first of all prove the following lemma:

Lemma 2.3. If $u$ is a weak solution of (EP), then we have

$$
\begin{aligned}
\int_{\mathbb{Q}} H_{0}(u-k)\left\{(h-H(k)) \xi_{x}-f \xi\right\} d x d t-\int_{\mathbb{Q}}(b(u)-b(k))^{+} \xi_{t} d x d t \\
\quad-\int_{I}\left(v_{0}-b(k)\right)^{+} \xi(0) d x=-\lim _{\epsilon \rightarrow 0} \int_{\mathbb{Q}}(h-H(k)) H_{\epsilon}(\varphi(u)-\varphi(k))_{x} \xi d x d t
\end{aligned}
$$

for any $(k, \xi) \in \mathbb{R} \times D([0, T) \times \bar{I})$ such that $\varphi(k) \notin E, k \geq 0$, and $\xi \geq 0$ and for any $(k, \xi) \in \mathbb{R} \times D([0, T) \times I)$ such that $\varphi(k) \notin E$ and $\xi \geq 0$; moreover,

$$
\begin{aligned}
\int_{\mathbb{Q}} H_{0}( & k-u)\left\{(h-H(k)) \xi_{x}-f \xi\right\} d x d t-\int_{\mathbb{Q}}(b(k)-b(u))^{+} \xi_{t} d x d t \\
& +\int_{I}\left(b(k)-v_{0}\right)^{+} \xi(0) d x=-\lim _{\epsilon \rightarrow 0} \int_{\mathbb{Q}}(h-H(k)) H_{\epsilon}(\varphi(k)-\varphi(u))_{x} \xi d x d t
\end{aligned}
$$

for any $(k, \xi) \in \mathbb{R} \times D([0, T) \times \bar{I})$ such that $\varphi(k) \notin E, k \leq 0$ and $\xi \geq 0$ and for any $(k, \xi) \in \mathbb{R} \times D([0, T) \times I)$ such that $\varphi(k) \notin E$ and $\xi \geq 0$, where

$$
E=\left\{r \in \operatorname{Im}(\varphi) \mid\left(\varphi^{-1}\right)_{0} \text { is discontinuous into } r\right\} \text {. }
$$


Remark 2.4. When the vector field $a(k, \xi)$ is of the type $a(k, \xi)=\xi_{x}+\phi(k)$, the right-hand sides of (5) and (6) have constant sign and therefore, the proof of Kato's inequalities is simpler (see [11, Lemmas 1 and 5]). When the nonlinearities are more general, as in this paper, the right-hand sides of (5) and (6) are not of constant sign, Carrillo's method does not apply directly and we need some extra effort to get Kato's inequalities (see the proofs of Theorem 2.2 and Theorem 2.5 below).

Proof of Lemma 2.3. We observe that for all $k$ such that $\varphi(k) \notin E$, we have

$$
H_{0}(u-k)=H_{0}(\varphi(u)-\varphi(k)) \text { on } \mathbb{Q} \text {. }
$$

Also,

$$
H_{\epsilon}(\varphi(u)-\varphi(k)) \xi \in L^{2}\left(0, T ; H_{0}^{1}(I)\right)
$$

since $\varphi(u) \in L^{2}(\mathbb{Q})$ and $\varphi(u(t)) \in H_{0}^{1}(I)$. Now, put

$$
\psi_{\epsilon}(z)=H_{\epsilon}(z-\varphi(s)) \quad \text { and } \quad B_{\psi_{\epsilon}}(z)=\int_{0}^{z} H_{\epsilon}\left(\varphi \circ\left(\left(b^{-1}\right)_{0}(r)\right)-\varphi(k)\right) d r .
$$

Since $\psi_{\epsilon}$ is bounded, we have

$$
B_{\psi_{\epsilon}}\left(v_{0}\right) \in L^{1}(I), \quad B_{\psi_{\epsilon}}(b(u)) \in L^{\infty}\left((0, T) ; L^{1}(I)\right)
$$

and

$$
\int_{\mathbb{Q}} B_{\psi_{\epsilon}}(b(u)) \xi_{t} d x d t+\int_{I} B_{\psi_{\epsilon}}\left(v_{0}\right) \xi(0) d x=-\int_{0}^{T}\left\langle b(u)_{t}, H_{\epsilon}(\varphi(u)-\varphi(k)) \xi\right\rangle d t .
$$

Moreover, since $u$ is a weak solution and $H_{\epsilon}(\varphi(u)-\varphi(k)) \xi \in L^{2}\left((0, T) ; H_{0}^{1}(I)\right)$, then

$$
\begin{aligned}
-\int_{0}^{T}\left\langle b(u)_{t},\right. & \left.H_{\epsilon}(\varphi(u)-\varphi(k)) \xi\right\rangle d t \\
& =\int_{\mathbb{Q}}\left\{(h-H(k))\left[H_{\epsilon}(\varphi(u)-\varphi(k)) \xi\right]_{x}-f H_{\epsilon}(\varphi(u)-\varphi(k)) \xi\right\} d x d t .
\end{aligned}
$$

This equality gives

$$
\begin{aligned}
\int_{\mathbb{Q}} B_{\psi_{\epsilon}}(b(u)) \xi_{t} d x d t & +\int_{I} B_{\psi_{\epsilon}}\left(v_{0}\right) \xi(0) d x \\
& =\int_{\mathbb{Q}}\left\{(h-H(k))\left[H_{\epsilon}(\varphi(u)-\varphi(k)) \xi\right]_{x}-f H_{\epsilon}(\varphi(u)-\varphi(k)) \xi\right\} d x d t
\end{aligned}
$$

In order to get equality (5), it is enough to show that

$$
\begin{aligned}
\lim _{\epsilon \rightarrow 0}\left(\int_{\mathbb{Q}} B_{\psi_{\epsilon}}(b(u)) \xi_{t} d x d t\right. & \left.+\int_{I} B_{\psi_{\epsilon}}\left(v_{0}\right) \xi(0) d x\right) \\
& =\int_{\mathbb{Q}}(b(u)-b(k))^{+} \xi_{t} d x d t+\int_{I}\left(v_{0}-b(k)\right)^{+} \xi(0) d x,
\end{aligned}
$$


for any $(k, \xi) \in D([0, T) \times \bar{I})$ such that $\varphi(k) \notin E, k \geq 0$, and $\xi \geq 0$ and for any $(k, \xi) \in \mathbb{R} \times D([0, T) \times I)$ such that $\varphi(k) \notin E$ and $\xi \geq 0$, where $B_{\psi_{\epsilon}}(b(u))$ is defined by

$$
B_{\psi_{\epsilon}}(b(u))=\int_{0}^{b(u)} H_{\epsilon}\left(\varphi \circ\left(\left(b^{-1}\right)_{0}(r)\right)-\varphi(k)\right) d r .
$$

- Step 1. For $k \geq 0$.

$$
B_{\psi_{\epsilon}}(b(u))=\int_{b(k)}^{b(u)} H_{\epsilon}\left(\varphi \circ\left(\left(b^{-1}\right)_{0}(r)\right)-\varphi(k)\right) d r \longrightarrow(b(u)-b(k))^{+} \quad \text { as } \epsilon \rightarrow 0 .
$$

Since $b$ is continuous, and $\varphi(k) \notin E$, we have that $\varphi \circ\left(\left(b^{-1}\right)_{0}(r)\right)-\varphi(k)>0, \forall r>b(k)$ and then

$$
H_{\epsilon}\left(\varphi \circ\left(\left(b^{-1}\right)_{0}(r)\right)-\varphi(k)\right) d r \longrightarrow 1 \quad \text { as } \epsilon \rightarrow 0 \quad \forall r>b(k) .
$$

Thus, in a similar way, we obtain

$$
\lim _{\epsilon \rightarrow 0} B_{\psi_{\epsilon}}\left(v_{0}\right)=\left(v_{0}-b(k)\right)^{+} .
$$

It is clear that $\left|B_{\psi_{\epsilon}}(b(u))\right| \leq|b(u)|$ and $\left|B_{\psi_{\epsilon}}\left(v_{0}\right)\right| \leq\left|v_{0}\right|$, which implies that

$$
\begin{aligned}
\lim _{\epsilon \rightarrow 0}\left(\int_{\mathbb{Q}} B_{\psi_{\epsilon}}(b(u)) \xi_{t} d x d t+\right. & \left.\int_{I} B_{\psi_{\epsilon}}\left(v_{0}\right) \xi(0) d x\right) \\
& =\int_{\mathbb{Q}}(b(u)-b(k))^{+} \xi_{t} d x d t+\int_{I}\left(v_{0}-b(k)\right)^{+} \xi(0) d x .
\end{aligned}
$$

- Step 2. For $k \leq 0$.

$$
\begin{aligned}
& B_{\psi_{\epsilon}}(b(u)) \\
& \quad=\int_{0}^{b(k)} H_{\epsilon}\left(\varphi \circ\left(\left(b^{-1}\right)_{0}(r)\right)-\varphi(k)\right) d r+\int_{b(k)}^{b(u)} H_{\epsilon}\left(\varphi \circ\left(\left(b^{-1}\right)_{0}(r)\right)-\varphi(k)\right) d r .
\end{aligned}
$$

Therefore,

$$
\lim _{\epsilon \rightarrow 0} B_{\psi_{\epsilon}}(b(u))=(b(u)-b(k))^{+}+b(k)
$$

and, in a similar way,

$$
\lim _{\epsilon \rightarrow 0} B_{\psi_{\epsilon}}\left(v_{0}\right)=\left(v_{0}-b(k)\right)^{+}+b(k) .
$$

Consequently,

$$
\begin{aligned}
\lim _{\epsilon \rightarrow 0}\left(\int_{\mathbb{Q}} B_{\psi_{\epsilon}}(b(u)) \xi_{t} d x d t\right. & \left.+\int_{I} B_{\psi_{\epsilon}}\left(v_{0}\right) \xi(0) d x\right)=\int_{\mathbb{Q}}(b(u)-b(k))^{+} \xi_{t} d x d t \\
& +\int_{\mathbb{Q}} b(k) \xi_{t} d x d t+\int_{I}\left(v_{0}-b(k)\right)^{+} \xi(0) d x+\int_{I} b(k) \xi(0) d x .
\end{aligned}
$$


Since

$$
\int_{\mathbb{Q}} b(k) \xi_{t} d x d t+\int_{I} b(k) \xi(0) d x=\int_{I} b(k)\left(\int_{0}^{T} \xi_{t} d t\right) d x+\int_{I} b(k) \xi(0) d x=0,
$$

then "

$$
\begin{aligned}
\lim _{\epsilon \rightarrow 0}\left(\int_{\mathbb{Q}} B_{\psi_{\epsilon}}(b(u)) \xi_{t} d x d t+\right. & \left.\int_{I} B_{\psi_{\epsilon}}\left(v_{0}\right) \xi(0) d x\right) \\
& =\int_{\mathbb{Q}}(b(u)-b(k))^{+} \xi_{t} d x d t+\int_{I}\left(v_{0}-b(k)\right)^{+} \xi(0) d x .
\end{aligned}
$$

Hence, (8) is established.

Again, taking limit as $\epsilon \rightarrow 0$ in (7) and using (8), we obtain

$$
\begin{aligned}
\int_{\mathbb{Q}}(b(u)-b(k))^{+} \xi_{t} d x d t & +\int_{I}\left(v_{0}-b(k)\right)^{+} \xi(0) d x \\
=\int_{\mathbb{Q}} H_{0}(u-k)\left[(h-H(k)) \xi_{x}-f \xi\right] d x d t & +\lim _{\epsilon \rightarrow 0} \int_{\mathbb{Q}}(h-H(k)) H_{\epsilon}(\varphi(u)-\varphi(k))_{x} \xi d x d t,
\end{aligned}
$$

from which we deduce (5). The inequality (6) is obtained in a similar way.

Next, we give the proof of Theorem 2.2.

Proof of Theorem 2.2. To do this, we use the method of doubling variables introduced by Kruzkhov for scalar conservation laws (see [16]).

Let $(s, y)$ and $(t, x)$ be two different pairs of variables in $\mathbb{Q}$. We set $u_{1}=u_{1}(s, y)$, $f_{1}=f_{1}(s, y), v_{0_{1}}=v_{0_{1}}(y)$ and $u_{2}=u_{2}(t, x), f_{2}=f_{2}(t, x), v_{0_{2}}=v_{0_{2}}(x)$.

Let $\xi$ be a smooth nonnegative function such that

$$
\begin{array}{ll}
(s, y) \longmapsto \xi(t, x, s, y) \in D^{+}([0, T) \times I) & \forall(t, x) \in \mathbb{Q}, \\
(t, x) \longmapsto \xi(t, x, s, y) \in D^{+}([0, T) \times I) & \forall(s, y) \in \mathbb{Q} .
\end{array}
$$

Let us define

$$
\mathbb{Q}_{1}=\left\{(s, y) \in \mathbb{Q} \mid \varphi\left(u_{1}(s, y)\right) \in E\right\}
$$

and

$$
\mathbb{Q}_{2}=\left\{(t, x) \in \mathbb{Q} \mid \varphi\left(u_{2}(t, x)\right) \in E\right\} .
$$

We deduce that

$$
\begin{array}{ll}
\varphi\left(u_{1}\right)_{y}=0 & \text { on } \mathbb{Q}_{1}, \\
\varphi\left(u_{2}\right)_{x}=0 & \text { on } \mathbb{Q}_{2} .
\end{array}
$$


Moreover, we easily check that

$$
H_{0}\left(u_{1}-u_{2}\right)=H_{0}\left(\varphi\left(u_{1}\right)-\varphi\left(u_{2}\right)\right) \text { in }\left[\mathbb{Q} \times\left(\mathbb{Q} \backslash \mathbb{Q}_{1}\right)\right] \cup\left[\left(\mathbb{Q} \backslash \mathbb{Q}_{2}\right) \times \mathbb{Q}\right]
$$

Replace $u$ by $u_{1}$ and $k$ by $u_{2}$ in $(5)$ and integrate over $\mathbb{Q} \backslash \mathbb{Q}_{2}$. Also replace $u$ by $u_{1}$ and $k$ by $u_{2}$ in (2) and integrate over $\mathbb{Q}_{2}$. Then, adding the two inequalities, we obtain

$$
\begin{aligned}
& \int_{\mathbb{Q} \times \mathbb{Q}} H_{0}\left(u_{1}-u_{2}\right)\left\{h_{1}\left(\xi_{y}+\xi_{x}\right)-\left(b\left(u_{1}\right)-b\left(u_{2}\right)\right) \xi_{s}-f_{1} \xi\right\} d y d s d x d t \\
& \quad-\int_{\mathbb{Q} \times I}\left(v_{0_{1}}-b\left(u_{2}\right)\right)+\xi(0) d y d x d t \\
& \leq \int_{\mathbb{Q} \times \mathbb{Q}} H_{0}\left(u_{1}-u_{2}\right) a\left(u_{2}, 0\right) \xi_{y} d y d s d x d t \\
&+\int_{\mathbb{Q} \times \mathbb{Q}} H_{0}\left(u_{1}-u_{2}\right) h_{1} \xi_{x} d y d s d x d t \\
&-\lim _{\epsilon \rightarrow 0} \int_{\left(\mathbb{Q}_{\mathbb{Q}} \mathbb{Q}_{2}\right) \times \mathbb{Q}}\left(h_{1}-a\left(u_{2}, 0\right)\right) H_{\epsilon}\left(\varphi\left(u_{1}\right)-\varphi\left(u_{2}\right)\right)_{y} \xi d y d s d x d t
\end{aligned}
$$

In the same way, we replace $k$ by $u_{1}$ and $u$ by $u_{2}$ in (6) and integrate over $\mathbb{Q} \backslash \mathbb{Q}_{1}$. Furthermore, replace $k$ by $u_{1}$ and $u$ by $u_{2}$ in (3) and integrate over $\mathbb{Q}_{1}$. Again, adding the two inequalities gives

$$
\begin{aligned}
\int_{\mathbb{Q} \times \mathbb{Q}} H_{0}\left(u_{1}\right. & \left.-u_{2}\right)\left\{h_{2}\left(\xi_{x}+\xi_{y}\right)-\left(b\left(u_{2}\right)-b\left(u_{1}\right)\right) \xi_{t}-f_{2} \xi\right\} d y d s d x d t \\
& +\int_{I \times \mathbb{Q}}\left(b\left(u_{1}\right)-v_{0_{2}}\right)^{+} \xi(0) d y d s d x \\
\geq & \int_{\mathbb{Q} \times \mathbb{Q}} H_{0}\left(u_{1}-u_{2}\right) a\left(u_{1}, 0\right) \xi_{x} d y d s d x d t \\
& +\int_{\mathbb{Q} \times \mathbb{Q}} H_{0}\left(u_{1}-u_{2}\right) h_{2} \xi_{y} d y d s d x d t \\
& -\lim _{\epsilon \rightarrow 0} \int_{\mathbb{Q} \times\left(\mathbb{Q} \backslash \mathbb{Q}_{1}\right)}\left(h_{2}-a\left(u_{1}, 0\right)\right) H_{\epsilon}\left(\varphi\left(u_{1}\right)-\varphi\left(u_{2}\right)\right)_{x} \xi d y d s d x d t
\end{aligned}
$$


Subtracting (13) from (12) gives

$$
\begin{aligned}
& \int_{\mathbb{Q} \times \mathbb{Q}} H_{0}\left(u_{1}-u_{2}\right) \\
& \quad \times\left\{\left(h_{1}-h_{2}\right)\left(\xi_{x}+\xi_{y}\right)+\left(b\left(u_{2}\right)-b\left(u_{1}\right)\right)\left(\xi_{s}+\xi_{t}\right)+\left(f_{2}-f_{1}\right) \xi\right\} d y d s d x d t \\
& \quad-\int_{\mathbb{Q} \times I}\left(v_{0_{1}}-b\left(u_{2}\right)\right)^{+} \xi(0) d y d x d t-\int_{I \times \mathbb{Q}}\left(b\left(u_{1}\right)-v_{0_{2}}\right)^{+} \xi(0) d y d s d x \\
& \leq \int_{\mathbb{Q} \times \mathbb{Q}} H_{0}\left(u_{1}-u_{2}\right)\left[h_{1}-a\left(u_{1}, 0\right)\right] \xi_{x} d y d s d x d t \\
& \quad-\int_{\mathbb{Q} \times \mathbb{Q}} H_{0}\left(u_{1}-u_{2}\right)\left[h_{2}-a\left(u_{2}, 0\right)\right] \xi_{y} d y d s d x d t \\
& -\lim _{\epsilon \rightarrow 0} \int_{\left(\mathbb{Q} \backslash \mathbb{Q}_{2}\right) \times \mathbb{Q}}\left[h_{1}-a\left(u_{2}, 0\right)\right] H_{\epsilon}\left(\varphi\left(u_{1}\right)-\varphi\left(u_{2}\right)\right)_{y} \xi d y d s d x d t \\
& +\lim _{\epsilon \rightarrow 0} \int_{\mathbb{Q} \times\left(\mathbb{Q} \backslash \mathbb{Q}_{1}\right)}\left[h_{2}-a\left(u_{1}, 0\right)\right] H_{\epsilon}\left(\varphi\left(u_{1}\right)-\varphi\left(u_{2}\right)\right)_{x} \xi d y d s d x d t .
\end{aligned}
$$

Using (10) and (11), we obtain

$$
\begin{aligned}
\int_{\mathbb{Q} \times \mathbb{Q}} H_{0}\left(u_{1}-u_{2}\right)\left[h_{1}-a\left(u_{1}, 0\right)\right] \xi_{x} d y d s d x d t \\
=\int_{\mathbb{Q} \times\left(\mathbb{Q} \backslash \mathbb{Q}_{1}\right)} H_{0}\left(u_{1}-u_{2}\right)\left[h_{1}-a\left(u_{1}, 0\right)\right] \xi_{x} \\
=\lim _{\epsilon \rightarrow 0} \int_{\mathbb{Q} \times\left(\mathbb{Q} \backslash \mathbb{Q}_{1}\right)}\left[h_{1}-a\left(u_{1}, 0\right)\right] H_{\epsilon}\left(\varphi\left(u_{1}\right)-\varphi\left(u_{2}\right)\right) \xi_{x} d y d s d x d t \\
=-\lim _{\epsilon \rightarrow 0} \int_{\mathbb{Q} \times\left(\mathbb{Q}_{\mathbb{Q}}\right)}\left[h_{1}-a\left(u_{1}, 0\right)\right] H_{\epsilon}\left(\varphi\left(u_{1}\right)-\varphi\left(u_{2}\right)\right)_{x} \xi d y d s d x d t
\end{aligned}
$$

and

$$
\begin{aligned}
\int_{\mathbb{Q} \times \mathbb{Q}} H_{0}\left(u_{1}-u_{2}\right)\left[h_{2}-a\left(u_{2}, 0\right)\right] \xi_{y} d y d s d x d t \\
=\int_{\left(\mathbb{Q} \backslash \mathbb{Q}_{2}\right) \times \mathbb{Q}} H_{0}\left(u_{1}-u_{2}\right)\left[h_{2}-a\left(u_{2}, 0\right)\right] \xi_{y} \\
=\lim _{\epsilon \rightarrow 0} \int_{\left(\mathbb{Q} \backslash \mathbb{Q}_{2}\right) \times \mathbb{Q}}\left[h_{2}-a\left(u_{2}, 0\right)\right] H_{\epsilon}\left(\varphi\left(u_{1}\right)-\varphi\left(u_{2}\right)\right) \xi_{y} d y d s d x d t \\
=-\lim _{\epsilon \rightarrow 0} \int_{\left(\mathbb{Q} \backslash \mathbb{Q}_{2}\right) \times \mathbb{Q}}\left[h_{2}-a\left(u_{2}, 0\right)\right] H_{\epsilon}\left(\varphi\left(u_{1}\right)-\varphi\left(u_{2}\right)\right)_{y} \xi d y d s d x d t .
\end{aligned}
$$


Substituting (15) and (16) in (14) and using (10), we obtain

$$
\begin{aligned}
\int_{\mathbb{Q} \times \mathbb{Q}} & H_{0}\left(u_{1}-u_{2}\right)\left\{\left(h_{1}-h_{2}\right)\left(\xi_{x}+\xi_{y}\right)+\left(b\left(u_{2}\right)-b\left(u_{1}\right)\right)\left(\xi_{s}+\xi_{t}\right)+\left(f_{2}-f_{1}\right) \xi\right\} d y d s d x d t \\
& -\int_{\mathbb{Q} \times I}\left(v_{0_{1}}-b\left(u_{2}\right)\right)^{+} \xi(0) d y d x d t-\int_{I \times \mathbb{Q}}\left(b\left(u_{1}\right)-v_{0_{2}}\right)^{+} \xi(0) d y d s d x \\
\leq & \lim _{\epsilon \rightarrow 0} \int_{\left(\mathbb{Q} \backslash \mathbb{Q}_{2}\right) \times\left(\mathbb{Q} \backslash \mathbb{Q}_{1}\right)}\left[h_{2}-h_{1}\right] \operatorname{div} H_{\epsilon}\left(\varphi\left(u_{1}\right)-\varphi\left(u_{2}\right)\right) \xi d y d s d x d t .
\end{aligned}
$$

Now, put

$$
I=\lim _{\epsilon \rightarrow 0} \int_{\left(\mathbb{Q} \backslash \mathbb{Q}_{2}\right) \times\left(\mathbb{Q} \mid \mathbb{Q}_{1}\right)}\left[h_{2}-h_{1}\right] \operatorname{div} H_{\epsilon}\left(\varphi\left(u_{1}\right)-\varphi\left(u_{2}\right)\right) \xi d y d s d x d t .
$$

Then, by $\left(\mathrm{H}_{2}\right)$,

$$
\begin{aligned}
I= & -\lim _{\epsilon \rightarrow 0} \int_{\left(\mathbb{Q} \mid \mathbb{Q}_{2}\right) \times\left(\mathbb{Q} \mid \mathbb{Q}_{1}\right)}\left[a\left(u_{1}, \varphi\left(u_{1}\right)_{y}\right)-a\left(u_{2}, \varphi\left(u_{2}\right)_{x}\right)\right] \\
& \times\left(\varphi\left(u_{1}\right)_{y}-\varphi\left(u_{2}\right)_{x}\right) H_{\epsilon}^{\prime}\left(\varphi\left(u_{1}\right)-\varphi\left(u_{2}\right)\right) \xi d y d s d x d t \\
\leq & \lim _{\epsilon \rightarrow 0} \int_{\left(\mathbb{Q} \mid \mathbb{Q}_{2}\right) \times\left(\mathbb{Q} \mid \mathbb{Q}_{1}\right)} M\left(u_{1}, u_{2}\right)\left(1+\left|\varphi\left(u_{1}\right)_{y}\right|^{2}+\left|\varphi\left(u_{2}\right)_{x}\right|^{2}\right) \\
& \times\left|\varphi\left(u_{1}\right)-\varphi\left(u_{2}\right)\right| H_{\epsilon}^{\prime}\left(\varphi\left(u_{1}\right)-\varphi\left(u_{2}\right)\right) \xi d y d s d x d t \\
- & \lim _{\epsilon \rightarrow 0} \int_{\left(\mathbb{Q} \mid \mathbb{Q}_{2}\right) \times\left(\mathbb{Q} \mid \mathbb{Q}_{1}\right)} \Gamma\left(\varphi\left(u_{1}\right), \varphi\left(u_{2}\right)\right) \varphi\left(u_{1}\right)_{y} H_{\epsilon}^{\prime}\left(\varphi\left(u_{1}\right)-\varphi\left(u_{2}\right)\right) \xi d y d s d x d t \\
& -\lim _{\epsilon \rightarrow 0} \int_{\left(\mathbb{Q} \mid \mathbb{Q}_{2}\right) \times\left(\mathbb{Q} \backslash \mathbb{Q}_{1}\right)} \hat{\Gamma}\left(\varphi\left(u_{1}\right), \varphi\left(u_{2}\right)\right) \varphi\left(u_{2}\right)_{x} H_{\epsilon}^{\prime}\left(\varphi\left(u_{1}\right)-\varphi\left(u_{2}\right)\right) \xi d y d s d x d t \\
= & \lim _{\epsilon \rightarrow 0} I_{1}-\lim _{\epsilon \rightarrow 0} I_{2}-\lim _{\epsilon \rightarrow 0} I_{3} .
\end{aligned}
$$

It is easy to see that $\lim _{\epsilon \rightarrow 0} I_{1}=0$.

Set

$$
F_{\epsilon}(z)=\int_{0}^{z} \Gamma\left(r, \varphi\left(u_{2}\right)\right) H_{\epsilon}^{\prime}\left(r-\varphi\left(u_{2}\right)\right) d r .
$$

We then have that

$$
\begin{aligned}
I_{2} & =\int_{\left(\mathbb{Q} \backslash \mathbb{Q}_{2}\right) \times\left(\mathbb{Q} \backslash \mathbb{Q}_{1}\right)} \operatorname{div}_{y} F_{\epsilon}\left(\varphi\left(u_{1}\right)\right) \xi d y d s d x d t \\
& =-\int_{\left(\mathbb{Q} \backslash \mathbb{Q}_{2}\right) \times\left(\mathbb{Q}_{\mathbb{Q}}\right)} F_{\epsilon}\left(\varphi\left(u_{1}\right)\right) \xi_{y} d y d s d x d t .
\end{aligned}
$$

Note that

$$
F_{\epsilon}(z)=\frac{1}{\epsilon} \int_{\min \left(z, \varphi\left(u_{2}\right)\right)}^{\min \left(z, \varphi\left(u_{2}\right)+\epsilon\right)} \Gamma\left(r, \varphi\left(u_{2}\right)\right) d r
$$


The function $\Gamma$ is in $C\left(\mathbb{R}^{2}\right)$ and attains its maximum and minimum on any compact subset of $\mathbb{R}$; in particular on $\left[\varphi\left(u_{2}\right), \varphi\left(u_{2}\right)+\epsilon\right]$, since $\left\|u_{2}\right\|_{\infty}$ is finite (see [19, Proposition 9]).

Again, there exist $m_{\epsilon}$ and $M_{\epsilon}$ such that

$$
m_{\epsilon} \leq \frac{1}{\epsilon} \int_{\min \left(z, \varphi\left(u_{2}\right)\right)}^{\min \left(z, \varphi\left(u_{2}\right)+\epsilon\right)} \Gamma\left(r, \varphi\left(u_{2}\right)\right) d r \leq M_{\epsilon} .
$$

By the intermediate value theorem, there exist $r_{1}(\epsilon)$ and $r_{2}(\epsilon)$ in $\left[\varphi\left(u_{2}\right), \varphi\left(u_{2}\right)+\epsilon\right]$ such that

$$
m_{\epsilon}=\Gamma\left(r_{1}(\epsilon), \varphi\left(u_{2}\right)\right)
$$

and

$$
M_{\epsilon}=\Gamma\left(r_{2}(\epsilon), \varphi\left(u_{2}\right)\right) .
$$

Since $r_{1}(\epsilon)$ and $r_{2}(\epsilon)$ are in $\left[\varphi\left(u_{2}\right), \varphi\left(u_{2}\right)+\epsilon\right]$, there exist $\theta_{1}$ and $\theta_{2}$ in $] 0,1[$ such that

$$
r_{1}(\epsilon)=\theta_{1}\left(\varphi\left(u_{2}\right)\right)+\left(1-\theta_{1}\right)\left(\varphi\left(u_{2}\right)+\epsilon\right)
$$

and

$$
r_{2}(\epsilon)=\theta_{2}\left(\varphi\left(u_{2}\right)\right)+\left(1-\theta_{2}\right)\left(\varphi\left(u_{2}\right)+\epsilon\right) .
$$

Consequently,

$$
\lim _{\epsilon \rightarrow 0} r_{1}(\epsilon)=\varphi\left(u_{2}\right) \quad \text { and } \quad \lim _{\epsilon \rightarrow 0} r_{2}(\epsilon)=\varphi\left(u_{2}\right) .
$$

Thus, we obtain

$$
\lim _{\epsilon \rightarrow 0} m_{\epsilon}=\Gamma\left(\varphi\left(u_{2}\right), \varphi\left(u_{2}\right)\right)=0 \quad \text { and } \quad \lim _{\epsilon \rightarrow 0} M_{\epsilon}=\Gamma\left(\varphi\left(u_{2}\right), \varphi\left(u_{2}\right)\right)=0 .
$$

This implies that $F_{\epsilon} \rightarrow 0$ as $\epsilon \rightarrow 0$, and so $\lim _{\epsilon \rightarrow 0} I_{2}=0$. Similarly, we get that $\lim _{\epsilon \rightarrow 0} I_{3}=0$. Consequently, $I \leq 0$ and, from (17), we deduce the following inequality:

$$
\begin{aligned}
& \int_{\mathbb{Q} \times \mathbb{Q}} H_{0}\left(u_{1}-u_{2}\right) \\
& \quad \times\left\{\left(h_{1}-h_{2}\right)\left(\xi_{x}+\xi_{y}\right)+\left(b\left(u_{2}\right)-b\left(u_{1}\right)\right)\left(\xi_{s}+\xi_{t}\right)+\left(f_{2}-f_{1}\right) \xi\right\} d y d s d x d t \\
& \quad-\int_{\mathbb{Q} \times I}\left(v_{0_{1}}-b\left(u_{2}\right)\right)^{+} \xi(0) d y d x d t-\int_{I \times \mathbb{Q}}\left(b\left(u_{1}\right)-v_{0_{2}}\right)^{+} \xi(0) d y d s d x \leq 0,
\end{aligned}
$$

for any nonnegative function $\xi$ satisfying (9).

Now let $\xi \in D([0, T) \times I)$ such that $\xi \geq 0$; let $\left(\rho_{n}\right)$ and $\left(\rho_{l}\right)$ be classical sequences of mollifiers in $\mathbb{R}$ such that $\rho_{l}(s)=\rho_{l}(-s)$ and $\rho_{n}(s)=\rho_{n}(-s)$. 
Define

$$
\xi^{l, n}(t, x, s, y)=\xi\left(\frac{t+s}{2}, \frac{x+y}{2}\right) \rho_{n}\left(\frac{x-y}{2}\right) \rho_{l}\left(\frac{t-s}{2}\right)
$$

Then $\xi^{l, n}$ are nonnegative functions satisfying (9) for $n$ and $l$ large enough.

By (18), for $n$ and $l$ large enough, we have

$$
\begin{aligned}
\int_{\mathbb{Q} \times \mathbb{Q}} H_{0}\left(u_{1}-u_{2}\right)\{( & \left(h_{1}-h_{2}\right)\left(\xi_{x}+\xi_{y}\right) \\
& \left.+\left(b\left(u_{2}\right)-b\left(u_{1}\right)\right)\left(\xi_{s}+\xi_{t}\right)+\left(f_{2}-f_{1}\right) \xi\right\} \rho_{n} \rho_{l} d y d s d x d t \\
& -\int_{\mathbb{Q} \times(\{0\} \times I)}\left(v_{0_{1}}-b\left(u_{2}\right)\right)^{+} \xi \rho_{n} \rho_{l} d y d x d t \\
& -\int_{(\{0\} \times I) \times \mathbb{Q}}\left(b\left(u_{1}\right)-v_{0_{2}}\right)^{+} \xi \rho_{n} \rho_{l} d y d s d x \leq 0 .
\end{aligned}
$$

Set

$$
\varphi^{(l)}(t, x, y)=\int_{t}^{T} \xi\left(\frac{r}{2}, \frac{x+y}{2}\right) \rho_{l}\left(\frac{r}{2}\right) d r=\int_{\min \left(t, \frac{1}{l}\right)}^{\frac{1}{l}} \xi\left(\frac{r}{2}, \frac{x+y}{2}\right) \rho_{l}\left(\frac{r}{2}\right) d r
$$

since $\operatorname{supp}\left(\rho_{l}\right) \subset\left(-\frac{1}{l},+\frac{1}{l}\right)$.

Since $u_{2}$ is an entropy solution, we replace $u$ by $u_{2}, k$ by $u_{1}(0, \cdot)$, and $\xi$ by $\rho_{n} \varphi^{(l)}$ in (3) and integrate over $I$ to obtain

$$
\begin{aligned}
-\int_{\mathbb{Q} \times(\{0\} \times I)} & \left(v_{0_{1}}-b\left(u_{2}\right)\right)^{+} \xi \rho_{n} \rho_{l} d y d x d t \\
= & \int_{\mathbb{Q} \times(\{0\} \times I)}\left(v_{0_{1}}-b\left(u_{2}\right)\right)^{+} \rho_{n} \varphi_{t}^{(l)} d y d x d t \\
\geq & -\int_{\mathbb{Q} \times(\{0\} \times I)} H_{0}\left(u_{1}(0, \cdot)-u_{2}\right) \\
& \times\left\{\left(a\left(u_{2}, \varphi\left(u_{2}\right)_{x}\right)-a\left(u_{1}(0, \cdot), 0\right)\right)\left(\rho_{n} \varphi^{(l)}\right)_{x}-f_{2} \rho_{n} \varphi^{(l)}\right\} d y d x d t \\
& -\int_{(\{0\} \times I) \times(\{0\} \times I)}\left(v_{0_{1}}-v_{0_{2}}\right)^{+} \rho_{n} \varphi^{(l)} d y d x,
\end{aligned}
$$


and since $\varphi^{(l)}=0$ when $t \geq \frac{1}{l}$, we have

$$
\begin{aligned}
-\int_{\mathbb{Q} \times(\{0\} \times I)} & \left(v_{0_{1}}-b\left(u_{2}\right)\right)^{+} \xi \rho_{n} \rho_{l} d y d x d t \\
\geq & -\int_{(\{0\} \times I) \times(\{0\} \times I)}\left(v_{0_{1}}-v_{0_{2}}\right)^{+} \rho_{n} \varphi^{(l)} d y d x \\
& -\int_{(\{0\} \times I) \times\left(\left(0, \frac{1}{l}\right) \times I\right)} H_{0}\left(u_{1}(0, \cdot)-u_{2}\right) \\
& \times\left\{\left(a\left(u_{2}, \varphi\left(u_{2}\right)_{x}\right)-a\left(u_{1}(0, \cdot), 0\right)\right)\left(\rho_{n} \varphi^{(l)}\right)_{x}-f_{2} \rho_{n} \varphi^{(l)}\right\} d y d x d t .
\end{aligned}
$$

It is easy to see that the second integral on the right hand side of inequality (20) converges to 0 when $l \rightarrow+\infty$. Moreover, since $\rho_{l}(s)=\rho_{l}(-s)$ for any $s \in \mathbb{R}$, then

$$
\lim _{l \rightarrow+\infty} \varphi^{l}(0, x, y)=\xi\left(0, \frac{x+y}{2}\right) \lim _{l \rightarrow+\infty} \int_{0}^{T} \rho_{l}(r) d r=\frac{\xi\left(0, \frac{x+y}{2}\right)}{2}
$$

for any $(x, y) \in I \times I$. Since $\varphi^{(l)}(0, x, y)$ is uniformly bounded in $L^{\infty}(I) \times L^{\infty}(I)$, we deduce that the first integral on the right hand side of inequality (20) converges to

$$
\frac{1}{2} \int_{(\{0\} \times I) \times(\{0\} \times I)}\left(v_{0_{1}}-v_{0_{2}}\right)^{+} \rho_{n} \xi d y d x,
$$

as $l \rightarrow+\infty$. Then we conclude that

$$
\begin{aligned}
-\limsup _{n \rightarrow+\infty} \limsup _{l \rightarrow+\infty} \int_{\mathbb{Q} \times(\{0\} \times I)} & \left(v_{0_{1}}-b\left(u_{2}\right)\right)^{+} \xi \rho_{n} \rho_{l} d y d x d t \\
\geq & -\lim _{n \rightarrow+\infty} \frac{1}{2} \int_{(\{0\} \times I) \times(\{0\} \times I)}\left(v_{0_{1}}-v_{0_{2}}\right)^{+} \rho_{n} \xi d y d x \\
= & -\frac{1}{2} \int_{\{0\} \times I}\left(v_{0_{1}}-v_{0_{2}}\right)^{+} \xi d x .
\end{aligned}
$$

Similarly, by considering the function

$$
\tilde{\varphi}^{(l)}(s, x, y)=\int_{s}^{T} \xi\left(\frac{r}{2}, \frac{x+y}{2}\right) \rho_{l}\left(\frac{-r}{2}\right) d r=\int_{\min \left(s, \frac{1}{\tau}\right)}^{\frac{1}{l}} \xi\left(\frac{r}{2}, \frac{x+y}{2}\right) \rho_{l}\left(\frac{-r}{2}\right) d r
$$

and the fact that $u_{1}$ is an entropy solution and letting $u=u_{1}, k=u_{2}(0, \cdot), \xi=\rho_{n} \tilde{\varphi}^{(l)}$ 
in (2), we deduce that

$$
\begin{aligned}
-\limsup _{n \rightarrow+\infty} \limsup _{l \rightarrow+\infty} \int_{(\{0\} \times I) \times \mathbb{Q}}\left(b\left(u_{1}\right)-v_{0_{2}}\right)^{+} \xi \rho_{n} \rho_{l} d y d s d x \\
\geq-\lim _{n \rightarrow+\infty} \frac{1}{2} \int_{(\{0\} \times I) \times(\{0\} \times I)}\left(v_{0_{1}}-v_{0_{2}}\right)^{+} \rho_{n} \xi d y d x \\
=-\frac{1}{2} \int_{\{0\} \times I}\left(v_{0_{1}}-v_{0_{2}}\right)^{+} \xi d x .
\end{aligned}
$$

Finally, taking limit as $n \rightarrow+\infty$ and $l \rightarrow+\infty$ in (19), and using (21) and (22), we get (4). This completes the proof.

Theorem 2.5 (Kato's Inequality). For $i=1,2$, let $f_{i} \in L^{2}\left((0, T) ; H^{-1}(I)\right) \cap L^{1}(\mathbb{Q})$ and $v_{0_{i}} \in L^{1}(I)$, let $u_{i}$ be an entropy solution of $(\mathrm{EP})$ with respect to data $\left(f_{i}, v_{0_{i}}\right)$. Then,

$$
\begin{aligned}
\int_{\mathbb{Q}} H_{0}\left(u_{1}-u_{2}\right)( & \left.h_{1}-h_{2}\right) \xi_{x} d x d t-\int_{\mathbb{Q}}\left(b\left(u_{1}\right)-b\left(u_{2}\right)\right)^{+} \xi_{t} d x d t \\
& -\int_{I}\left(v_{0_{1}}-v_{0_{2}}\right)^{+} \xi(0) d x \leq \int_{\mathbb{Q}} H_{0}\left(u_{1}-u_{2}\right)\left(f_{1}-f_{2}\right) \xi d x d t
\end{aligned}
$$

for any nonnegative $\xi \in D([0, T) \times \bar{I})$.

Proof. As in the proof of the previous theorem we consider two different pairs of variables $(s, y)$ and $(t, x)$ in $\mathbb{Q}$, and we assume that $u_{1}=u_{1}(s, y), f_{1}=f_{1}(s, y)$, $v_{0_{1}}=v_{0_{1}}(y)$, and that $u_{2}=u_{2}(t, x), f_{2}=f_{2}(t, x), v_{0_{2}}=v_{0_{2}}(x)$. Let $\mathbb{Q}_{1}$ and $\mathbb{Q}_{2}$ be defined as in the proof of the previous theorem.

Let $\xi=\xi(t, x, s, y)$ be a nonnegative and smooth function in $\mathbb{R}^{4}$ such that

$$
\begin{array}{ll}
(s, y) \longmapsto \xi(t, x, s, y) \in D([0, T) \times \bar{I}) & \text { for any }(t, x) \in \mathbb{Q}, \\
(t, x) \longmapsto \xi(t, x, s, y) \in D([0, T) \times I) & \text { for any }(s, y) \in \mathbb{Q} .
\end{array}
$$

Then, replace $u$ by $u_{1}$ and $k$ by $u_{2}^{+}$in (5) and integrate over $\mathbb{Q} \backslash \mathbb{Q}_{2}^{+}$. Also replace $u$ by $u_{1}$ and $k$ by $u_{2}^{+}$in (2) and integrate over $\mathbb{Q}_{2}^{+}$. Then, according to (10) and (11) 
by adding the two inequalities, we obtain

$$
\begin{aligned}
\int_{\mathbb{Q} \times \mathbb{Q}} H_{0}\left(u_{1}-u_{2}^{+}\right) & \left\{h_{1}\left(\xi_{x}+\xi_{y}\right)+\left(b\left(u_{2}^{+}\right)-b\left(u_{1}\right)\right) \xi_{s}-f_{1} \xi\right\} d y d s d x d t \\
& -\int_{\mathbb{Q} \times I}\left(v_{0_{1}}-b\left(u_{2}^{+}\right)\right)^{+} \xi(0) d y d x d t \\
\leq & -\lim _{\epsilon \rightarrow 0} \int_{\left(\mathbb{Q} \backslash \mathbb{Q}_{2}^{+}\right) \times \mathbb{Q}}\left(h_{1}-a\left(u_{2}^{+}, 0\right)\right) H_{\epsilon}\left(\varphi\left(u_{1}\right)-\varphi\left(u_{2}^{+}\right)\right)_{y} \xi d y d s d x d t \\
& -\lim _{\epsilon \rightarrow 0} \int_{\mathbb{Q} \times \mathbb{Q}}\left(h_{1}-a\left(u_{1}, 0\right)\right) H_{\epsilon}\left(\varphi\left(u_{1}\right)-\varphi\left(u_{2}^{+}\right)\right)_{x} \xi d y d s d x d t \\
& +\int_{\mathbb{Q} \times \mathbb{Q}} H_{0}\left(u_{1}-u_{2}^{+}\right) a\left(u_{1}, 0\right) \xi_{x} d y d s d x d t \\
& +\int_{\mathbb{Q} \times \mathbb{Q}} H_{0}\left(u_{1}-u_{2}^{+}\right) a\left(u_{2}^{+}, 0\right) \xi_{y} d y d s d x d t .
\end{aligned}
$$

Since we integrate where $u_{1}$ and $v_{0_{1}}$ are positive, the above inequality can be written as

$$
\begin{aligned}
\int_{\mathbb{Q} \times \mathbb{Q}} H_{0}\left(u_{1}^{+}-u_{2}^{+}\right)\left\{h_{1}^{+}\left(\xi_{x}+\xi_{y}\right)+\left(b\left(u_{2}^{+}\right)-b\left(u_{1}^{+}\right)\right) \xi_{s}-f_{1} \xi\right\} d y d s d x d t \\
\quad-\int_{\mathbb{Q} \times I}\left(v_{0_{1}}^{+}-b\left(u_{2}^{+}\right)\right)^{+} \xi(0) d y d x d t \\
\leq-\lim _{\epsilon \rightarrow 0} \int_{\left(\mathbb{Q} \backslash \mathbb{Q}_{2}^{+}\right) \times \mathbb{Q}}\left(h_{1}^{+}-a\left(u_{2}^{+}, 0\right)\right) H_{\epsilon}\left(\varphi\left(u_{1}^{+}\right)-\varphi\left(u_{2}^{+}\right)\right)_{y} \xi d y d s d x d t \\
\quad-\lim _{\epsilon \rightarrow 0} \int_{\mathbb{Q} \times\left(\mathbb{Q} \backslash \mathbb{Q}_{1}^{+}\right)}\left(h_{1}^{+}-a\left(u_{1}^{+}, 0\right)\right) H_{\epsilon}\left(\varphi\left(u_{1}^{+}\right)-\varphi\left(u_{2}^{+}\right)\right)_{x} \xi d y d s d x d t \\
+\int_{\mathbb{Q} \times \mathbb{Q}} H_{0}\left(u_{1}^{+}-u_{2}^{+}\right) a\left(u_{1}^{+}, 0\right) \xi_{x} d y d s d x d t \\
+\int_{\mathbb{Q} \times \mathbb{Q}} H_{0}\left(u_{1}^{+}-u_{2}^{+}\right) a\left(u_{2}^{+}, 0\right) \xi_{y} d y d s d x d t .
\end{aligned}
$$

where $h_{1}^{+}=a\left(u_{1}^{+}, \varphi\left(u_{1}^{+}\right)_{x}\right)$ and $\mathbb{Q}_{1}^{+}=\left\{(s, y) \in \mathbb{Q} / \varphi\left(u_{1}^{+}(s, y)\right) \in E\right\}$.

Recall that, by $(24),(t, x) \mapsto \xi(t, x, s, y) \in D([0, T) \times I)$ for any $(s, y) \in \mathbb{Q}$; then, we replace $u$ by $u_{2}$ and $k$ by $u_{1}^{+}$in (6) and integrate over $\mathbb{Q} \backslash \mathbb{Q}_{1}^{+}$. Also replace $u$ by $u_{2}$ and $k$ by $u_{1}^{+}$in (3) and integrate over $\mathbb{Q}_{1}^{+}$. Then, adding the two inequalities, 
we obtain

$$
\begin{aligned}
\int_{\mathbb{Q} \times \mathbb{Q}} H_{0}\left(u_{1}^{+}-u_{2}\right)\left\{\left(h_{2}-a\left(u_{1}^{+}, 0\right)\right) \xi_{x}+\left(b\left(u_{1}^{+}\right)-b\left(u_{2}\right)\right) \xi_{t}-f_{2} \xi\right\} d y d s d x d t \\
\quad+\int_{I \times \mathbb{Q}}\left(b\left(u_{1}^{+}\right)-v_{0_{2}}\right)^{+} \xi(0) d y d s d x \\
\geq-\lim _{\epsilon \rightarrow 0} \int_{\mathbb{Q} \times\left(\mathbb{Q} \backslash \mathbb{Q}_{1}^{+}\right)}\left(h_{2}-a\left(u_{1}^{+}, 0\right)\right) H_{\epsilon}\left(\varphi\left(u_{1}^{+}\right)-\varphi\left(u_{2}\right)\right)_{x} \xi d y d s d x d t
\end{aligned}
$$

Note that

$$
H_{0}\left(u_{1}^{+}-u_{2}\right)=H_{0}\left(u_{1}^{+}-u_{2}^{+}\right)\left(1-H_{0}\left(u_{2}^{-}\right)\right)+H_{0}\left(u_{2}^{-}\right) .
$$

Then we get from (26) that

$$
\begin{aligned}
\int_{\mathbb{Q} \times \mathbb{Q}} H_{0}( & \left.u_{1}^{+}-u_{2}^{+}\right) \\
& \times\left\{\left(h_{2}^{+}-a\left(u_{1}^{+}, 0\right)\right) \xi_{x}+\left(b\left(u_{1}^{+}\right)-b\left(u_{2}^{+}\right)\right) \xi_{t}-\left(1-H_{0}\left(u_{2}^{-}\right)\right) f_{2} \xi\right\} d y d s d x d t \\
& +\int_{I \times \mathbb{Q}}\left(b\left(u_{1}^{+}\right)-v_{0_{2}}^{+}\right)^{+} \xi(0) d y d s d x-\int_{I \times \mathbb{Q}} v_{0_{2}}^{-} \xi(0) d y d s d x \\
& +\int_{\mathbb{Q} \times \mathbb{Q}} H_{0}\left(u_{2}^{-}\right)\left\{h_{2} \xi_{x}-b\left(u_{2}\right) \xi_{t}-f_{2} \xi\right\} d y d s d x d t \\
\geq & -\lim _{\epsilon \rightarrow 0} \int_{\mathbb{Q} \times\left(\mathbb{Q} \backslash \mathbb{Q}_{1}^{+}\right)}\left(h_{2}-a\left(u_{1}^{+}, 0\right)\right) H_{\epsilon}\left(\varphi\left(u_{1}^{+}\right)-\varphi\left(u_{2}\right)\right)_{x} \xi d y d s d x d t
\end{aligned}
$$

where $h_{2}^{+}=a\left(u_{2}^{+}, \varphi\left(u_{2}^{+}\right)_{x}\right)$.

Moreover, since for almost every $(t, x) \in \mathbb{Q}$ the function

$$
(s, y) \longmapsto H_{\epsilon}\left(\varphi\left(u_{1}^{+}\right)-\varphi\left(u_{2}^{+}\right)\right) \xi(t, x, s, y)
$$

belongs to $L^{2}\left((0, T) ; H_{0}^{1}(I)\right)$, we have

$$
\int_{\mathbb{Q} \times \mathbb{Q}}\left(h_{2}^{+}-a\left(u_{2}^{+}, 0\right)\right)\left(H_{\epsilon}\left(\varphi\left(u_{1}^{+}\right)-\varphi\left(u_{2}^{+}\right)\right) \xi\right)_{y} d y d s d x d t=0
$$

and, therefore, by taking into account (10) and (11),

$$
\begin{aligned}
\lim _{\epsilon \rightarrow 0} \int_{\mathbb{Q} \times \mathbb{Q}}\left(h_{2}^{+}-a\right. & \left.\left(u_{2}^{+}, 0\right)\right) H_{\epsilon}\left(\varphi\left(u_{1}^{+}\right)-\varphi\left(u_{2}^{+}\right)\right)_{y} \xi d y d s d x d t \\
& =-\lim _{\epsilon \rightarrow 0} \int_{\mathbb{Q} \times \mathbb{Q}}\left(h_{2}^{+}-a\left(u_{2}^{+}, 0\right)\right) H_{\epsilon}\left(\varphi\left(u_{1}^{+}\right)-\varphi\left(u_{2}^{+}\right)\right) \xi_{y} d y d s d x d t \\
& =-\int_{\mathbb{Q} \times \mathbb{Q}}\left(h_{2}^{+}-a\left(u_{2}^{+}, 0\right)\right) H_{0}\left(\varphi\left(u_{1}^{+}\right)-\varphi\left(u_{2}^{+}\right)\right) \xi_{y} d y d s d x d t \\
& =-\int_{\left(\mathbb{Q} \backslash \mathbb{Q}_{2}^{+}\right) \times \mathbb{Q}}\left(h_{2}^{+}-a\left(u_{2}^{+}, 0\right)\right) H_{0}\left(u_{1}^{+}-u_{2}^{+}\right) \xi_{y} d y d s d x d t=
\end{aligned}
$$




$$
=-\int_{\mathbb{Q} \times \mathbb{Q}} H_{0}\left(u_{1}^{+}-u_{2}^{+}\right)\left(h_{2}^{+}-a\left(u_{2}^{+}, 0\right)\right) \xi_{y} d y d s d x d t .
$$

From inequality (27) we deduce that

$$
\begin{aligned}
\int_{\mathbb{Q} \times \mathbb{Q}} H_{0} & \left(u_{1}^{+}-u_{2}^{+}\right) \\
& \times\left\{h_{2}^{+}\left(\xi_{x}+\xi_{y}\right)+\left(b\left(u_{1}^{+}\right)-b\left(u_{2}^{+}\right)\right) \xi_{t}-\left(1-H_{0}\left(u_{2}^{-}\right)\right) f_{2} \xi\right\} d y d s d x d t \\
& +\int_{I \times \mathbb{Q}}\left(b\left(u_{1}^{+}\right)-v_{0_{2}}^{+}\right)^{+} \xi(0) d y d s d x-\int_{I \times \mathbb{Q}} v_{0_{2}}^{-} \xi(0) d y d s d x \\
& +\int_{\mathbb{Q} \times \mathbb{Q}} H_{0}\left(u_{2}^{-}\right)\left\{h_{2} \xi_{x}-b\left(u_{2}\right) \xi_{t}-f_{2} \xi\right\} d y d s d x d t \\
\geq & -\lim _{\epsilon \rightarrow 0} \int_{\mathbb{Q} \times\left(\mathbb{Q} \mid \mathbb{Q}_{1}^{+}\right)}\left(h_{2}-a\left(u_{1}^{+}, 0\right)\right) H_{\epsilon}\left(\varphi\left(u_{1}^{+}\right)-\varphi\left(u_{2}\right)\right)_{x} \xi d y d s d x d t \\
& -\lim _{\epsilon \rightarrow 0} \int_{\left(\mathbb{Q} \mid \mathbb{Q}_{2}^{+}\right) \times \mathbb{Q}}\left(h_{2}^{+}-a\left(u_{2}^{+}, 0\right)\right) H_{\epsilon}\left(\varphi\left(u_{1}^{+}\right)-\varphi\left(u_{2}^{+}\right)\right)_{y} \xi d y d s d x d t \\
& +\int_{\mathbb{Q} \times \mathbb{Q}} H_{0}\left(u_{1}^{+}-u_{2}^{+}\right) a\left(u_{1}^{+}, 0\right) \xi_{x} d y d s d x d t \\
& +\int_{\mathbb{Q} \times \mathbb{Q}} H_{0}\left(u_{1}^{+}-u_{2}^{+}\right) a\left(u_{2}^{+}, 0\right) \xi_{y} d y d s d x d t .
\end{aligned}
$$

Now, subtracting (28) from (25), we get

$$
\begin{aligned}
& \int_{\mathbb{Q} \times \mathbb{Q}} H_{0}\left(u_{1}^{+}-u_{2}^{+}\right)\left\{\left(h_{1}^{+}-h_{2}^{+}\right)\left(\xi_{x}+\xi_{y}\right)+\left(b\left(u_{2}^{+}\right)-b\left(u_{1}^{+}\right)\right)\left(\xi_{s}+\xi_{t}\right)\right. \\
& \left.-\left(f_{1}-\left(1-H_{0}\left(u_{2}^{-}\right)\right) f_{2}\right) \xi\right\} d y d s d x d t \\
& \quad-\int_{\mathbb{Q} \times I}\left(v_{0_{1}}^{+}-b\left(u_{2}^{+}\right)\right)^{+} \xi(0) d y d x d t-\int_{I \times \mathbb{Q}}\left(b\left(u_{1}^{+}\right)-v_{0_{2}}^{+}\right)^{+} \xi(0) d y d s d x \\
& \quad-\int_{\mathbb{Q} \times \mathbb{Q}} H_{0}\left(u_{2}^{-}\right)\left\{h_{2} \xi_{x}-b\left(u_{2}\right) \xi_{t}-f_{2} \xi\right\} d y d s d x d t+\int_{I \times \mathbb{Q}} v_{0_{2}}^{-} \xi(0) d y d s d x \\
& \leq-\lim _{\epsilon \rightarrow 0} \int_{\left(\mathbb{Q} \mid \mathbb{Q}_{2}^{+}\right) \times \mathbb{Q}}\left(h_{1}^{+}-a\left(u_{2}^{+}, 0\right)\right) H_{\epsilon}\left(\varphi\left(u_{1}^{+}\right)-\varphi\left(u_{2}^{+}\right)\right)_{y} \xi d y d s d x d t \\
& \quad-\lim _{\epsilon \rightarrow 0} \int_{\mathbb{Q} \times\left(\mathbb{Q} \backslash \mathbb{Q}_{1}^{+}\right)}\left(h_{1}^{+}-a\left(u_{1}^{+}, 0\right)\right) H_{\epsilon}\left(\varphi\left(u_{1}^{+}\right)-\varphi\left(u_{2}^{+}\right)\right)_{x} \xi d y d s d x d t \\
& +\lim _{\epsilon \rightarrow 0} \int_{\mathbb{Q} \times\left(\mathbb{Q} \backslash \mathbb{Q}_{1}^{+}\right)}\left(h_{2}-a\left(u_{1}^{+}, 0\right)\right) H_{\epsilon}\left(\varphi\left(u_{1}^{+}\right)-\varphi\left(u_{2}\right)\right)_{x} \xi d y d s d x d t \\
& +\lim _{\epsilon \rightarrow 0} \int_{\left(\mathbb{Q} \backslash \mathbb{Q}_{2}^{+}\right) \times \mathbb{Q}}\left(h_{2}^{+}-a\left(u_{2}^{+}, 0\right)\right) H_{\epsilon}\left(\varphi\left(u_{1}^{+}\right)-\varphi\left(u_{2}^{+}\right)\right)_{y} \xi d y d s d x d t .
\end{aligned}
$$


We know that

$$
\begin{aligned}
\lim _{\epsilon \rightarrow 0} \int_{\mathbb{Q} \times\left(\mathbb{Q} \backslash \mathbb{Q}_{1}^{+}\right)}\left(h_{2}-a\left(u_{1}^{+}, 0\right)\right) H_{\epsilon}\left(\varphi\left(u_{1}^{+}\right)-\varphi\left(u_{2}\right)\right)_{x} \xi d y d s d x d t \\
=\lim _{\epsilon \rightarrow 0} \int_{\mathbb{Q} \times\left(\mathbb{Q} \backslash \mathbb{Q}_{1}^{+}\right)} H_{0}\left(u_{2}^{-}\right)\left(h_{2}-a\left(u_{1}^{+}, 0\right)\right) H_{\epsilon}\left(\varphi\left(u_{1}^{+}\right)-\varphi\left(u_{2}\right)\right)_{x} \xi d y d s d x d t \\
\quad+\lim _{\epsilon \rightarrow 0} \int_{\mathbb{Q} \times\left(\mathbb{Q} \backslash \mathbb{Q}_{1}^{+}\right)}\left(h_{2}^{+}-a\left(u_{1}^{+}, 0\right)\right) H_{\epsilon}\left(\varphi\left(u_{1}^{+}\right)-\varphi\left(u_{2}^{+}\right)\right)_{x} \xi d y d s d x d t
\end{aligned}
$$

since $1=\left(1-H_{0}\left(u_{2}^{-}\right)\right)+H_{0}\left(u_{2}^{-}\right)$and $1-H_{0}\left(u_{2}^{-}\right)=0$ when $u_{2}<0$.

Using (30) in (29), it follows that

$$
\begin{aligned}
\int_{\mathbb{Q} \times \mathbb{Q}} & H_{0}\left(u_{1}^{+}-u_{2}^{+}\right)\left\{\left(h_{1}^{+}-h_{2}^{+}\right)\left(\xi_{x}+\xi_{y}\right)+\left(b\left(u_{2}^{+}\right)-b\left(u_{1}^{+}\right)\right)\left(\xi_{s}+\xi_{t}\right)\right. \\
- & \left.\left(f_{1}-\left(1-H_{0}\left(u_{2}^{-}\right)\right) f_{2}\right) \xi\right\} d y d s d x d t \\
& -\int_{\mathbb{Q} \times I}\left(v_{0_{1}}^{+}-b\left(u_{2}^{+}\right)\right)^{+} \xi(0) d y d x d t-\int_{I \times \mathbb{Q}}\left(b\left(u_{1}^{+}\right)-v_{0_{2}}^{+}\right)^{+} \xi(0) d y d s d x \\
& -\int_{\mathbb{Q} \times \mathbb{Q}} H_{0}\left(u_{2}^{-}\right)\left\{h_{2} \xi_{x}-b\left(u_{2}\right) \xi_{t}-f_{2} \xi\right\} d y d s d x d t+\int_{I \times \mathbb{Q}} v_{0_{2}}^{-} \xi(0) d y d s d x \\
\leq & \lim _{\epsilon \rightarrow 0} \int_{\mathbb{Q} \times\left(\mathbb{Q} \backslash \mathbb{Q}_{1}^{+}\right)} H_{0}\left(u_{2}^{-}\right)\left(h_{2}-a\left(u_{1}^{+}, 0\right)\right) H_{\epsilon}\left(\varphi\left(u_{1}^{+}\right)-\varphi\left(u_{2}\right)\right)_{x} \xi d y d s d x d t \\
& +\lim _{\epsilon \rightarrow 0} \int_{\left(\mathbb{Q} \backslash \mathbb{Q}_{2}^{+}\right) \times\left(\mathbb{Q} \backslash \mathbb{Q}_{1}^{+}\right)}\left[h_{2}^{+}-h_{1}^{+}\right] \operatorname{div} H_{\epsilon}\left(\varphi\left(u_{1}^{+}\right)-\varphi\left(u_{2}^{+}\right)\right) \xi d y d s d x d t .
\end{aligned}
$$

Define

$$
J=\lim _{\epsilon \rightarrow 0} \int_{\left(\mathbb{Q} \backslash \mathbb{Q}_{2}^{+}\right) \times\left(\mathbb{Q} \backslash \mathbb{Q}_{1}^{+}\right)}\left[h_{2}^{+}-h_{1}^{+}\right] \operatorname{div} H_{\epsilon}\left(\varphi\left(u_{1}^{+}\right)-\varphi\left(u_{2}^{+}\right)\right) \xi d y d s d x d t
$$

and

$$
K=\lim _{\epsilon \rightarrow 0} \int_{\mathbb{Q} \times\left(\mathbb{Q} \backslash \mathbb{Q}_{1}^{+}\right)} H_{0}\left(u_{2}^{-}\right)\left(h_{2}-a\left(u_{1}^{+}, 0\right)\right) H_{\epsilon}\left(\varphi\left(u_{1}^{+}\right)-\varphi\left(u_{2}\right)\right)_{x} \xi d y d s d x d t
$$

As in the proof of theorem 2.2 for $I \leq 0$, we prove that $J \leq 0$ and $K \leq 0$. Then we ob- 
tain from (31) that

$$
\begin{aligned}
\int_{\mathbb{Q} \times \mathbb{Q}} & H_{0}\left(u_{1}^{+}-u_{2}^{+}\right)\left\{\left(h_{1}^{+}-h_{2}^{+}\right)\left(\xi_{x}+\xi_{y}\right)+\left(b\left(u_{2}^{+}\right)-b\left(u_{1}^{+}\right)\right)\left(\xi_{s}+\xi_{t}\right)\right. \\
& \left.-\left(f_{1}-\left(1-H_{0}\left(u_{2}^{-}\right)\right) f_{2}\right) \xi\right\} d y d s d x d t \\
& -\int_{\mathbb{Q} \times I}\left(v_{0_{1}}^{+}-b\left(u_{2}^{+}\right)\right)^{+} \xi(0) d y d x d t-\int_{I \times \mathbb{Q}}\left(b\left(u_{1}^{+}\right)-v_{0_{2}}^{+}\right)^{+} \xi(0) d y d s d x \\
\leq & \int_{\mathbb{Q} \times \mathbb{Q}} H_{0}\left(u_{2}^{-}\right)\left\{h_{2} \xi_{x}-b\left(u_{2}\right) \xi_{t}-f_{2} \xi\right\} d y d s d x d t-\int_{I \times \mathbb{Q}} v_{0_{2}}^{-} \xi(0) d y d s d x
\end{aligned}
$$

Now, let $\xi \in D([0, T) \times \mathbb{R}), \xi \geq 0$, be such that

$$
\operatorname{supp}(\xi) \cap([0, T) \times \mathbb{R}) \subset[0, T) \times B,
$$

where $B$ is an interval for which either $B \cap \partial I=\emptyset$ or $B \subset \subset B^{\prime}$ is a part of the graph of a Lipschitz continuous function. Then there exists a sequence of mollifiers $\rho_{l}$ defined on $\mathbb{R}$, with $\operatorname{supp}\left(\rho_{l}\right) \subset(-2 / l, 0)$ and there exists a sequence of mollifiers $\rho_{n}$ defined on $\mathbb{R}$ such that, for $n$ large enough,

$$
\begin{gathered}
x \longmapsto \rho_{n}\left(\frac{x-y}{2}\right) \in D(I) \quad \forall y \in B, \\
\chi_{n}(x)=\int_{I} \rho_{n}\left(\frac{x-y}{2}\right) d y \text { is an increasing sequence for } x \in B, \\
\chi_{n}(x)=1 \text { for any } x \in B \text { such that } d(x, \mathbb{R} \backslash I)>c / n,
\end{gathered}
$$

where $c$ is a positive constant depending on $B$. Then, for $n$ and $l$ large enough, the function

$$
\xi^{(l, n)}(t, x, s, y)=\xi(t, x) \rho_{n}\left(\frac{x-y}{2}\right) \rho_{l}\left(\frac{t-s}{2}\right)
$$

satisfies (24) and the function

$$
\begin{aligned}
\xi^{(n)}(t, x) & =\int_{Q} \xi^{(l, n)}(t, x, s, y) d y d s \\
& =\xi(t, x) \int_{I} \rho_{n}\left(\frac{x-y}{2}\right) d y \int_{0}^{T} \rho_{l}\left(\frac{t-s}{2}\right) d s=\xi \chi_{n}
\end{aligned}
$$

satisfies

$$
\begin{gathered}
\xi^{(n)} \in D([0, T) \times I), \quad \xi^{(n)} \leq \xi^{\left(n^{\prime}\right)} \forall n^{\prime} \geq n, \\
\xi^{(n)}(t, x)=\xi(t, x) \quad \forall x \text { such that } d(x, \mathbb{R} \backslash I)>c / n,
\end{gathered}
$$

where $c$ is a positive constant depending on $B$. Obviously $\xi^{(n)} \leq \xi$, and $\xi^{(n)}$ converges to $\xi$ in $L^{r}(\mathbb{Q})$ for any $1 \leq r<+\infty$. 
Replace $\xi$ by $\xi^{(l, n)}$ in $(32)$ to get

$$
\begin{aligned}
\int_{\mathbb{Q} \times \mathbb{Q}} H_{0}\left(u_{1}^{+}-u_{2}^{+}\right)\{( & \left.h_{1}^{+}-h_{2}^{+}\right)\left(\xi_{x}^{(l, n)}+\xi_{y}^{(l, n)}\right)+\left(b\left(u_{2}^{+}\right)-b\left(u_{1}^{+}\right)\right)\left(\xi_{s}^{(l, n)}+\xi_{t}^{(l, n)}\right) \\
& \left.-\left(f_{1}-\left(1-H_{0}\left(u_{2}^{-}\right)\right) f_{2}\right) \xi^{(l, n)}\right\} d y d s d x d t \\
& -\int_{\mathbb{Q} \times(\{0\} \times I)}\left(v_{0_{1}}^{+}-b\left(u_{2}^{+}\right)\right)^{+} \xi^{(l, n)} d y d x d t \\
& -\int_{(\{0\} \times I) \times \mathbb{Q}}\left(b\left(u_{1}^{+}\right)-v_{0_{2}}^{+}\right)^{+} \xi^{(l, n)} d y d s d x \\
\leq & \int_{\mathbb{Q} \times \mathbb{Q}} H_{0}\left(u_{2}^{-}\right)\left\{h_{2} \xi_{x}^{(l, n)}-b\left(u_{2}\right) \xi_{t}^{(l, n)}-f_{2} \xi^{(l, n)}\right\} d y d s d x d t \\
& -\int_{(\{0\} \times I) \times \mathbb{Q}} v_{0_{2}}^{-} \xi^{(l, n)} d y d s d x .
\end{aligned}
$$

Therefore, since $\left(\partial_{x}+\partial_{y}\right)\left(\rho_{n}\left(\frac{x-y}{2}\right)\right)=\left(\partial_{t}+\partial_{s}\right)\left(\rho_{l}\left(\frac{t-s}{2}\right)\right)=0$, it follows from the above inequality that

$$
\begin{aligned}
\int_{\mathbb{Q} \times \mathbb{Q}} H_{0}\left(u_{1}^{+}-\right. & \left.u_{2}^{+}\right)\left\{\left(h_{1}^{+}-h_{2}^{+}\right) \xi_{x}+\left(b\left(u_{2}^{+}\right)-b\left(u_{1}^{+}\right)\right) \xi_{t}\right. \\
& \left.-\left(f_{1}-\left(1-H_{0}\left(u_{2}^{-}\right)\right) f_{2}\right) \xi\right\} \rho_{n} \rho_{l} d y d s d x d t \\
& -\int_{\mathbb{Q} \times(\{0\} \times I)}\left(v_{0_{1}}^{+}-b\left(u_{2}^{+}\right)\right)^{+} \xi \rho_{n} \rho_{l} d y d x d t \\
& -\int_{(\{0\} \times I) \times \mathbb{Q}}\left(b\left(u_{1}^{+}\right)-v_{0_{2}}^{+}\right)^{+} \xi \rho_{n} \rho_{l} d y d s d x \\
\leq & \int_{\mathbb{Q}} H_{0}\left(u_{2}^{-}\right)\left\{h_{2} \xi_{x}^{(n)}-b\left(u_{2}\right) \xi_{t}^{(n)}-f_{2} \xi^{(n)}\right\} d x d t-\int_{\{0\} \times I} v_{0_{2}}^{-} \xi^{(n)} d x .
\end{aligned}
$$

Since $\int_{\{0\} \times I} v_{0_{2}}^{-} \xi^{(n)} d x \geq 0$, we can write the inequality above as

$$
\begin{aligned}
\int_{\mathbb{Q} \times \mathbb{Q}} H_{0}\left(u_{1}^{+}-u_{2}^{+}\right)\left\{\left(h_{1}^{+}-h_{2}^{+}\right) \xi_{x}+\left(b\left(u_{2}^{+}\right)-b\left(u_{1}^{+}\right)\right) \xi_{t}\right. \\
\left.-\left(f_{1}-\left(1-H_{0}\left(u_{2}^{-}\right)\right) f_{2}\right) \xi\right\} \rho_{n} \rho_{l} d y d s d x d t \\
-\int_{\mathbb{Q} \times(\{0\} \times I)}\left(v_{0_{1}}^{+}-b\left(u_{2}^{+}\right)\right)^{+} \xi \rho_{n} \rho_{l} d y d x d t \\
-\int_{(\{0\} \times I) \times \mathbb{Q}}\left(b\left(u_{1}^{+}\right)-v_{0_{2}}^{+}\right)^{+} \xi \rho_{n} \rho_{l} d y d s d x \leq
\end{aligned}
$$




$$
\begin{aligned}
\leq & \int_{\{0\} \times I} v_{0_{2}}^{-} \xi^{(n)} d x+\int_{\mathbb{Q}} H_{0}\left(u_{2}^{-}\right)\left\{h_{2} \xi_{x}^{(n)}-b\left(u_{2}\right) \xi_{t}^{(n)}-f_{2} \xi^{(n)}\right\} d x d t \\
\Longleftrightarrow & I_{1}-I_{2}-I_{3} \leq I_{4}, \\
\lim _{n, l \rightarrow+\infty} I_{1}= & \int_{\mathbb{Q}} H_{0}\left(u_{1}^{+}-u_{2}^{+}\right) \\
& \times\left\{\left(h_{1}^{+}-h_{2}^{+}\right) \xi_{x}+\left(b\left(u_{2}^{+}\right)-b\left(u_{1}^{+}\right)\right) \xi_{t}-\left(f_{1}-\left(1-H_{0}\left(u_{2}^{-}\right)\right) f_{2}\right) \xi\right\} d x d t,
\end{aligned}
$$

where every function depends on $(t, x)$.

As in the proof of Theorem 2.2, we prove that

$$
-\limsup _{n \rightarrow+\infty} \limsup _{l \rightarrow+\infty} I_{2} \geq-\frac{1}{2} \int_{\{0\} \times I}\left(v_{0_{1}}^{+}-v_{0_{2}}^{+}\right)^{+} \xi d x
$$

and

$$
-\limsup _{n \rightarrow+\infty} \limsup _{l \rightarrow+\infty} I_{3} \geq-\frac{1}{2} \int_{\{0\} \times I}\left(v_{0_{1}}^{+}-v_{0_{2}}^{+}\right)^{+} \xi d x,
$$

where every function depends on $x$.

Since $u_{2}$ is an entropy solution,

$$
\eta \longmapsto L(\eta)=\int_{\{0\} \times I} v_{0_{2}}^{-} \xi d x+\int_{\mathbb{Q}} H_{0}\left(u_{2}^{-}\right)\left\{h_{2} \xi_{x}-b\left(u_{2}\right) \xi_{t}-f_{2} \xi\right\} d x d t
$$

is monotone increasing. In particular, since

$$
0 \leq \xi^{(n)} \leq \xi^{\left(n^{\prime}\right)} \leq \xi
$$

for any $n^{\prime}$ such that $n \leq n^{\prime}$, we deduce that $L\left(\xi^{(n)}\right)$ is an increasing sequence satisfying $0 \leq L\left(\xi^{(n)}\right) \leq L(\xi)$, and therefore it converges when $n \rightarrow+\infty$. Then

$$
\begin{aligned}
& \int_{\mathbb{Q}} H_{0}\left(u_{1}^{+}-u_{2}^{+}\right) \\
& \quad \times\left\{\left(h_{1}^{+}-h_{2}^{+}\right) \xi_{x}+\left(b\left(u_{2}^{+}\right)-b\left(u_{1}^{+}\right)\right) \xi_{t}-\left(f_{1}-\left(1-H_{0}\left(u_{2}^{-}\right)\right) f_{2}\right) \xi\right\} d x d t \\
& -\int_{\{0\} \times I}\left(v_{0_{1}}^{+}-v_{0_{2}}^{+}\right)^{+} \xi d x \\
& \leq \lim _{n \rightarrow+\infty}\left(\int_{\mathbb{Q}} H_{0}\left(u_{2}^{-}\right)\left\{h_{2} \xi_{x}^{(n)}-b\left(u_{2}\right) \xi_{t}^{(n)}-f_{2} \xi^{(n)}\right\} d x d t+\int_{\{0\} \times I} v_{0_{2}}^{-} \xi^{(n)} d x\right)
\end{aligned}
$$

for any nonnegative $\xi \in D([0, T) \times B)$.

Now, considering Remark 1.6, and replacing $u_{1}$ by $-u_{2}, u_{2}$ by $-u_{1}, f_{1}$ by $-f_{2}, f_{2}$ 
by $-f_{1}, b$ by $\tilde{b}, \varphi$ by $\tilde{\varphi}$, and $a$ by $\tilde{a}$, and arguing as above, we deduce that

$$
\begin{aligned}
& \int_{\mathbb{Q}} H_{0}\left(\left(-u_{1}^{-}\right)-\left(-u_{2}^{-}\right)\right) \\
& \left.\quad \times\left\{\left(h_{1}^{-}-h_{2}^{-}\right) \xi_{x}+\left(b\left(-u_{2}^{-}\right)-b\left(-u_{1}^{-}\right)\right) \xi_{t}-\left(1-H_{0}\left(u_{1}^{+}\right)\right) f_{1}-f_{2}\right) \xi\right\} d x d t \\
& -\int_{\{0\} \times I}\left(b\left(-u_{1}(0, \cdot)^{-}\right)-b\left(-u_{2}(0, \cdot)^{-}\right)\right)^{+} \xi d x \\
& \leq-\lim _{n \rightarrow+\infty}\left(\int_{\mathbb{Q}} H_{0}\left(u_{1}^{+}\right)\left\{h_{1} \xi_{x}^{(n)}-b\left(u_{1}\right) \xi_{t}^{(n)}-f_{1} \xi^{(n)}\right\} d x d t\right. \\
& \left.\quad-\int_{\{0\} \times I} v_{0_{1}}^{+} \xi^{(n)} d x\right)
\end{aligned}
$$

for any nonnegative $\xi \in D([0, T) \times B)$, where

$$
\begin{aligned}
h_{1}^{-}=a\left(-u_{1}^{-}, \varphi\left(-u_{1}^{-}\right)_{x}\right), & h_{2}^{-}=a\left(-u_{2}^{-}, \varphi\left(-u_{2}^{-}\right)_{x}\right), \\
v_{0}^{+}=b\left(u(0, \cdot)^{+}\right), & v_{0}^{-}=b\left(u(0, \cdot)^{-}\right) .
\end{aligned}
$$

It is easy to check that

$$
\begin{gathered}
H_{0}\left(\left(-u_{1}^{-}\right)-\left(-u_{2}^{-}\right)\right)\left(1-H_{0}\left(u_{1}^{+}\right)\right) H_{0}\left(u_{2}^{-}\right)+H_{0}\left(u_{1}^{+}-u_{2}^{+}\right) H_{0}\left(u_{1}^{+}\right) \\
=H_{0}\left(u_{1}-u_{2}\right),
\end{gathered} \begin{gathered}
H_{0}\left(\left(-u_{1}^{-}\right)-\left(-u_{2}^{-}\right)\right) H_{0}\left(u_{2}^{-}\right)+H_{0}\left(u_{1}^{+}-u_{2}^{+}\right)\left(1-H_{0}\left(u_{2}^{-}\right)\right) H_{0}\left(u_{1}^{+}\right) \\
=H_{0}\left(u_{1}-u_{2}\right), \\
H_{0}\left(u_{1}^{+}-u_{2}^{+}\right)=H_{0}\left(u_{1}^{+}-u_{2}^{+}\right) H_{0}\left(u_{1}^{+}\right), \\
H_{0}\left(\left(-u_{1}^{-}\right)-\left(-u_{2}^{-}\right)\right)=H_{0}\left(\left(-u_{1}^{-}\right)-\left(-u_{2}^{-}\right)\right) H_{0}\left(u_{2}^{-}\right), \\
H_{0}\left(u_{1}^{+}-u_{2}^{+}\right)\left(h_{1}^{+}-h_{2}^{+}\right)+H_{0}\left(\left(-u_{1}^{-}\right)-\left(-u_{2}^{-}\right)\right)\left(h_{1}^{-}-h_{2}^{-}\right) \\
=H_{0}\left(u_{1}-u_{2}\right)\left(h_{1}-h_{2}\right)
\end{gathered}
$$

and

$$
\begin{array}{r}
H_{0}\left(u_{1}^{+}-u_{2}^{+}\right)\left(b\left(u_{1}^{+}\right)-b\left(u_{2}^{+}\right)\right)+H_{0}\left(\left(-u_{1}^{-}\right)-\left(-u_{2}^{-}\right)\right)\left(b\left(-u_{1}^{-}\right)-b\left(-u_{2}^{-}\right)\right) \\
=H_{0}\left(u_{1}-u_{2}\right)\left(b\left(u_{1}\right)-b\left(u_{2}\right)\right) .
\end{array}
$$


Then adding (33) and (34) by using (35)-(40), we obtain

$$
\begin{aligned}
\int_{\mathbb{Q}} H_{0}\left(u_{1}-u_{2}\right)\left\{\left(h_{1}\right.\right. & \left.\left.-h_{2}\right) \xi_{x}+\left(b\left(u_{2}\right)-b\left(u_{1}\right)\right) \xi_{t}-\left(f_{1}-f_{2}\right) \xi\right\} d x d t \\
& -\int_{\{0\} \times I}\left(v_{0_{1}}-v_{0_{2}}\right)+\xi d x \\
\leq & \lim _{n \rightarrow+\infty}\left(\int_{\mathbb{Q}} H_{0}\left(u_{2}^{-}\right)\left\{h_{2} \xi_{x}^{(n)}-b\left(u_{2}\right) \xi_{t}^{(n)}-f_{2} \xi^{(n)}\right\} d x d t\right. \\
& \left.+\int_{\{0\} \times I} v_{0_{2}}^{-} \xi^{(n)} d x\right) \\
& -\lim _{n \rightarrow+\infty}\left(\int_{\mathbb{Q}} H_{0}\left(u_{1}^{+}\right)\left\{h_{1} \xi_{x}^{(n)}-b\left(u_{1}\right) \xi_{t}^{(n)}-f_{1} \xi^{(n)}\right\} d x d t\right. \\
& \left.-\int_{\{0\} \times I} v_{0_{1}}^{+} \xi^{(n)} d x\right),
\end{aligned}
$$

for any nonnegative $\xi \in D([0, T) \times B)$.

Now let $\xi \in D([0, T) \times B), \xi \geq 0$. Then $\xi^{\left(n^{\prime}\right)}=\xi \chi_{n^{\prime}} \in D([0, T) \times I)$ and by applying Theorem 2.2 , we have

$$
\begin{aligned}
& \int_{\mathbb{Q}} H_{0}\left(u_{1}-u_{2}\right) \\
& \quad \times\left\{\left(h_{1}-h_{2}\right)\left(\xi \chi_{n^{\prime}}\right)_{x}+\left(b\left(u_{2}\right)-b\left(u_{1}\right)\right)\left(\xi_{t} \chi_{n^{\prime}}\right)-\left(f_{1}-f_{2}\right) \xi \chi_{n^{\prime}}\right\} d x d t \\
& -\int_{I}\left(v_{0_{1}}-v_{0_{2}}\right)^{+} \xi(0) \chi_{n^{\prime}} d x \leq 0 .
\end{aligned}
$$

Therefore

$$
\begin{aligned}
\int_{\mathbb{Q}} H_{0}\left(u_{1}-u_{2}\right)\{( & \left.\left.h_{1}-h_{2}\right) \xi_{x}+\left(b\left(u_{2}\right)-b\left(u_{1}\right)\right) \xi_{t}-\left(f_{1}-f_{2}\right) \xi\right\} d x d t \\
& -\int_{I}\left(v_{0_{1}}-v_{0_{2}}\right)+\xi(0) d x \\
\leq & \int_{\mathbb{Q}} H_{0}\left(u_{1}-u_{2}\right)\left\{\left(h_{1}-h_{2}\right)\left(\xi\left(1-\chi_{n^{\prime}}\right)\right)_{x}\right. \\
& \left.+\left(b\left(u_{2}\right)-b\left(u_{1}\right)\right) \xi_{t}\left(1-\chi_{n^{\prime}}\right)-\left(f_{1}-f_{2}\right) \xi\left(1-\chi_{n^{\prime}}\right)\right\} d x d t \\
& -\int_{I}\left(v_{0_{1}}-v_{0_{2}}\right)^{+} \xi(0)\left(1-\chi_{n^{\prime}}\right) d x,
\end{aligned}
$$


and since $\xi\left(1-\chi_{n^{\prime}}\right)$ is a nonnegative function of $D([0, T) \times B)$, from $(41)$, we have

$$
\begin{aligned}
\int_{\mathbb{Q}} H_{0}\left(u_{1}-u_{2}\right)\{( & \left.h_{1}-h_{2}\right)\left(\xi\left(1-\chi_{n^{\prime}}\right)\right)_{x}+\left(b\left(u_{2}\right)-b\left(u_{1}\right)\right) \xi_{t}\left(1-\chi_{n^{\prime}}\right) \\
& \left.-\left(f_{1}-f_{2}\right) \xi\left(1-\chi_{n^{\prime}}\right)\right\} d x d t-\int_{I}\left(v_{0_{1}}-v_{0_{2}}\right)^{+} \xi(0)\left(1-\chi_{n^{\prime}}\right) d x \\
\leq & \lim _{n \rightarrow+\infty}\left[\int _ { \mathbb { Q } } H _ { 0 } ( u _ { 2 } ^ { - } ) \left\{h_{2}\left(\xi\left(1-\chi_{n^{\prime}}\right) \chi_{n}\right)_{x}\right.\right. \\
& \left.\left.-b\left(u_{2}\right) \xi_{t}\left(1-\chi_{n^{\prime}}\right) \chi_{n}\right)-f_{2} \xi\left(1-\chi_{n^{\prime}}\right) \chi_{n}\right\} d x d t \\
& \left.+\int_{I} v_{0_{2}}^{-} \xi(0)\left(1-\chi_{n^{\prime}}\right) \chi_{n} d x\right] \\
& -\lim _{n \rightarrow+\infty}\left[\int _ { \mathbb { Q } } H _ { 0 } ( u _ { 1 } ^ { + } ) \left\{h_{1}\left(\xi\left(1-\chi_{n^{\prime}}\right) \chi_{n}\right)_{x}\right.\right. \\
& \left.\left.-b\left(u_{1}\right) \xi_{t}\left(1-\chi_{n^{\prime}}\right) \chi_{n}\right)-f_{1} \xi\left(1-\chi_{n^{\prime}}\right) \chi_{n}\right\} d x d t \\
& \left.-\int_{I} v_{0_{1}}^{+} \xi(0)\left(1-\chi_{n^{\prime}}\right) \chi_{n} d x\right] .
\end{aligned}
$$

Moreover, for any $n^{\prime}$ there exists $n_{0}^{\prime}$ such that $\chi_{n}=1$ in $\operatorname{supp}\left(\chi_{n^{\prime}}\right)$ for $n>n_{0}^{\prime}$. Therefore,

$$
\begin{aligned}
\lim _{n^{\prime} \rightarrow+\infty} & \lim _{n \rightarrow+\infty}\left[\int _ { \mathbb { Q } } H _ { 0 } ( u _ { 2 } ^ { - } ) \left\{h_{2}\left(\xi\left(1-\chi_{n^{\prime}}\right) \chi_{n}\right)_{x}\right.\right. \\
& \left.\left.-b\left(u_{2}\right) \xi_{t}\left(1-\chi_{n^{\prime}}\right) \chi_{n}-f_{2} \xi\left(1-\chi_{n^{\prime}}\right) \chi_{n}\right\} d x d t+\int_{I} v_{0_{2}}^{-} \xi(0)\left(1-\chi_{n^{\prime}}\right) \chi_{n} d x\right] \\
& -\lim _{n^{\prime} \rightarrow+\infty} \lim _{n \rightarrow+\infty}\left[\int _ { \mathbb { Q } } H _ { 0 } ( u _ { 1 } ^ { + } ) \left\{h_{1}\left(\xi\left(1-\chi_{n^{\prime}}\right) \chi_{n}\right)_{x}\right.\right. \\
& \left.\left.-b\left(u_{1}\right) \xi_{t}\left(1-\chi_{n^{\prime}}\right) \chi_{n}-f_{1} \xi\left(1-\chi_{n^{\prime}}\right) \chi_{n}\right\} d x d t-\int_{I} v_{0_{1}}^{+} \xi(0)\left(1-\chi_{n^{\prime}}\right) \chi_{n} d x\right] \\
= & \lim _{n^{\prime} \rightarrow+\infty} \lim _{n \rightarrow+\infty}\left[\int _ { \mathbb { Q } } H _ { 0 } ( u _ { 2 } ^ { - } ) \left\{h_{2}\left(\xi\left(\chi_{n}-\chi_{n^{\prime}}\right)\right)_{x}\right.\right. \\
& \left.\left.-b\left(u_{2}\right) \xi_{t}\left(\chi_{n}-\chi_{n^{\prime}}\right)-f_{2} \xi\left(\chi_{n}-\chi_{n^{\prime}}\right)\right\} d x d t+\int_{I} v_{0_{2}}^{-} \xi(0)\left(\chi_{n}-\chi_{n^{\prime}}\right) d x\right] \\
& -\lim _{n^{\prime} \rightarrow+\infty} \lim _{n \rightarrow+\infty}\left[\int _ { \mathbb { Q } } H _ { 0 } ( u _ { 1 } ^ { + } ) \left\{h_{1}\left(\xi\left(\chi_{n}-\chi_{n^{\prime}}\right)\right)_{x}\right.\right. \\
& \left.\left.-b\left(u_{1}\right) \xi_{t}\left(\chi_{n}-\chi_{n^{\prime}}\right)-f_{1} \xi\left(\chi_{n}-\chi_{n^{\prime}}\right)\right\} d x d t-\int_{I} v_{0_{1}}^{+} \xi(0)\left(\chi_{n}-\chi_{n^{\prime}}\right) d x\right]=0
\end{aligned}
$$


and, by (42), we obtain

$$
\begin{aligned}
\int_{\mathbb{Q}} H_{0}\left(u_{1}-u_{2}\right)\left\{\left(h_{1}-h_{2}\right) \xi_{x}+\left(b\left(u_{2}\right)-b\left(u_{1}\right)\right) \xi_{t}\right. & \left.-\left(f_{1}-f_{2}\right) \xi\right\} d x d t \\
& -\int_{I}\left(v_{0_{1}}-v_{0_{2}}\right)^{+} \xi(0) d x \leq 0
\end{aligned}
$$

for any nonnegative $\xi \in D([0, T) \times B)$.

Now let $I_{0} \subset \subset I$ be such that $I_{0} \cup \bigcup_{i=1}^{k} B_{i}$ is a covering of $I$ where $B_{i}$, for $1 \leq i \leq k$, are intervals satisfying (36). Let $\left(\varphi_{i}\right)_{i=0}^{k}$ be a partition of unity related to the above covering $\left(\varphi_{0} \in D\left(I_{0}\right), \varphi_{i} \in D\left(B_{i}\right)\right.$, for $\left.1 \leq i \leq k\right)$. Let $\xi \in D([0, T) \times \bar{I})$, $\xi \geq 0$, and let $\xi_{i}=\xi \varphi_{i}$. Then, applying (4) for $i=0$ and (43) for $1 \leq i \leq k$, we have

$$
\begin{aligned}
\int_{\mathbb{Q}} H_{0}\left(u_{1}-u_{2}\right)\left\{\left(h_{1}-h_{2}\right) \xi_{i_{x}}+\left(b\left(u_{2}\right)-b\left(u_{1}\right)\right) \xi_{i_{t}}\right. & \left.-\left(f_{1}-f_{2}\right) \xi_{i}\right\} d x d t \\
& -\int_{I}\left(v_{0_{1}}-v_{0_{2}}\right)^{+} \xi_{i}(0) d x \leq 0
\end{aligned}
$$

for all $0 \leq i \leq k$.

Since $\xi=\sum_{i=0}^{k} \xi_{i}$, we have

$$
\begin{aligned}
\int_{\mathbb{Q}} H_{0}\left(u_{1}-u_{2}\right)\left\{\left(h_{1}-h_{2}\right) \xi_{x}+\left(b\left(u_{2}\right)-b\left(u_{1}\right)\right) \xi_{t}-\right. & \left.\left(f_{1}-f_{2}\right) \xi\right\} d x d t \\
& -\int_{I}\left(v_{0_{1}}-v_{0_{2}}\right)^{+} \xi(0) d x \leq 0
\end{aligned}
$$

for any nonnegative $\xi \in D([0, T) \times \bar{I})$. This completes the proof of Theorem 2.5.

As a consequence of Theorem 2.5, we have

Corollary 2.6. Let $v_{0_{i}} \in L^{1}(I)$, let $f_{i} \in L^{2}\left((0, T) ; H^{-1}(I)\right) \cap L^{1}(\mathbb{Q})$, and let $u_{i}$ be an entropy solution of $(\mathrm{EP})\left(f_{i}, v_{0_{i}}\right), i=1,2$. Then

$$
\begin{aligned}
& \int_{I}\left(b\left(u_{1}(t)\right)-b\left(u_{2}(t)\right)\right)^{+} d x \\
& \leq \int_{I}\left(v_{0_{1}}-v_{0_{2}}\right)^{+} d x+\int_{0}^{t} \int_{I} H_{0}\left(u_{1}-u_{2}\right)\left(f_{1}-f_{2}\right) d x d s
\end{aligned}
$$

and, therefore,

$$
\left\|b\left(u_{1}(t)\right)-b\left(u_{2}(t)\right)\right\|_{L^{1}(I)} \leq\left\|v_{0_{1}}-v_{0_{2}}\right\|_{L^{1}(I)}+\int_{0}^{t}\left\|f_{1}-f_{2}\right\|_{L^{1}(I)} d s .
$$


In particular, if $v_{0_{1}} \leq v_{0_{2}}$ almost everywhere in $I$ and $f_{1} \leq f_{2}$ almost everywhere in $\mathbb{Q}$, then

$$
b\left(u_{1}\right) \leq b\left(u_{2}\right) \quad \text { a.e. in } \mathbb{Q} .
$$

Moreover, if $f_{1}=f_{2}$ and $v_{0_{1}}=v_{0_{2}}$, then

$$
b\left(u_{1}\right)=b\left(u_{2}\right) .
$$

Proof. Let $\xi \in D([0, T) \times \bar{I})$ be such that $\xi \geq 0$ and $\xi=\xi(t)\left(\xi_{x} \equiv 0\right)$. From (23) we have

$$
\begin{aligned}
-\int_{0}^{T}\left(\int_{I}\left(b\left(u_{1}\right)-b\left(u_{2}\right)\right)^{+} d x\right) \xi_{t} d t- & \int_{I}\left(v_{0_{1}}-v_{0_{2}}\right)^{+} \xi(0) d x \\
& \leq \int_{0}^{T}\left(\int_{I} H_{0}\left(u_{1}-u_{2}\right)\left(f_{1}-f_{2}\right) d x\right) \xi d t
\end{aligned}
$$

and therefore

$$
\begin{aligned}
-\int_{0}^{T}\left(\int _ { I } \left[\left(b\left(u_{1}\right)-b\left(u_{2}\right)\right)^{+}-\left(v_{0_{1}}-\right.\right.\right. & \left.\left.\left.v_{0_{2}}\right)^{+}\right] d x\right) \xi_{t} d t \\
& \leq \int_{0}^{T}\left(\int_{I} H_{0}\left(u_{1}-u_{2}\right)\left(f_{1}-f_{2}\right) d x\right) \xi d t .
\end{aligned}
$$

Let us introduce the functions

$$
\begin{aligned}
& G(t)= \begin{cases}\int_{I}\left[\left(b\left(u_{1}(t)\right)-b\left(u_{2}(t)\right)\right)^{+}-\left(v_{0_{1}}-v_{0_{2}}\right)^{+}\right] d x & \text { for } t \in(0, T), \\
0 & \text { for } t \in(-T, 0),\end{cases} \\
& F(t)= \begin{cases}\int_{I} H_{0}\left(u_{1}(t)-u_{2}(t)\right)\left(f_{1}(t)-f_{2}(t)\right) d x & \text { for } t \in(0, T), \\
0 & \text { for } t \in(-T, 0) .\end{cases}
\end{aligned}
$$

Then, from (48), we deduce that

$$
\frac{d G}{d t} \leq F \quad \text { in } D^{\prime}(-T, T),
$$

and therefore, since $G$ and $F$ vanish for $t<0$, we have that

$$
G(t) \leq \int_{0}^{t} F(s) d s .
$$

Hence, we easily deduce (44). From (44), we easily deduce (45)-(47), which shows the corollary. 
Remark 2.7. As the reader can see, in the proof of the main theorem of this paper (see Theorem 2.5), and also in [18], assumptions $\left(\mathrm{H}_{3}\right)$ and $\left(\mathrm{H}_{4}\right)$ are not needed to obtain uniqueness of entropy solution of (EP) nor to define the operator $A_{b}$. They seem to be needed just to show existence of weak and entropy solutions (see [19]). Another interesting and difficult open question is to generalize the results to higher dimensions. Note that the techniques used in this paper can be employed to get uniqueness of entropy solutions in several dimensions space (cf. [3]), but it is not possible to generalize the techniques used in $[18,19]$ to get existence of entropy solutions in higher dimensions due to the fact that the main assumption for the proof of existence in the one dimension case is assumption $\left(\mathrm{H}_{1}\right)$ (see [18] for more details), which is equivalent to the coerciveness assumption only in dimension one but not equivalent to the coerciveness assumption in several dimensions.

\section{References}

[1] H. W. Alt and S. Luckhaus, Quasilinear elliptic-parabolic differential equations, Math. Z. 183 (1983), no. 3, 311-341.

[2] K. Ammar and P. Wittbold, Existence of renormalized solutions of degenerate elliptic-parabolic problems, Proc. Roy. Soc. Edinburgh Sect. A 133 (2003), no. 3, 477-496.

[3] B. Andreianov, M. Bendahmane, K. H. Karlsen, and S. Ouaro, Well-posedness result for triply nonlinear degenerate parabolic equations, in preparation.

[4] W. Arendt and Ph. Bénilan, Inegalités de Kato et semi-groupes sous-markoviens, Rev. Mat. Univ. Complut. Madrid 5 (1992), no. 2-3, 279-308 (French, with English summary).

[5] Ph. Bénilan, Equations d'évolution dans un espace de Banach quelconque et applications, Thèse d'état, Universite Pierre et Marie Curie (Paris VI), Orsay, 1972.

[6] Ph. Bénilan, M. G. Crandall, and A. Pazy, Evolution equation governed by accretive operators, book to appear.

[7] Ph. Bénilan and S. N. Kruzhkov, First-order quasilinear equations with continuous nonlinearities, Dokl. Akad. Nauk 339 (1994), no. 2, 151-154 (Russian); English transl., Russian Acad. Sci. Dokl. Math. 50 (1995), no. 3, 391-396.

[8] P. Bénilan and H. Touré, Sur l'équation générale $u_{t}=a\left(\cdot, u, \phi(\cdot, u)_{x}\right)_{x}+v$ dans $L^{1}$. II: $L e$ problème d'évolution, Ann. Inst. H. Poincaré Anal. Non Linéaire 12 (1995), no. 6, 727-761 (French, with English and French summaries).

[9] Ph. Bénilan and P. Wittbold, On mild and weak solutions of elliptic-parabolic problems, Adv. Differential Equations 1 (1996), no. 6, 1053-1073.

[10] _ Sur un problème parabolique-elliptique, M2AN Math. Model. Numer. Anal. 33 (1999), no. 1, 121-127 (French, with English and French summaries).

[11] J. Carrillo, Entropy solutions for nonlinear degenerate problems, Arch. Ration. Mech. Anal. 147 (1999), no. 4, 269-361.

[12] J. Carrillo and P. Wittbold, Uniqueness of renormalized solutions of degenerate elliptic-parabolic problems, J. Differential Equations 156 (1999), no. 1, 93-121.

[13] M. G. Crandall, The semigroup approach to first order quasilinear equations in several space variables, Israel J. Math. 12 (1972), 108-132.

[14] M. G. Crandall and T. M. Liggett, Generation of semi-groups of nonlinear transformations on general Banach spaces, Amer. J. Math. 93 (1971), 265-298. 
[15] J. I. Diaz and R. Kersner, On a nonlinear degenerate parabolic equation in infiltration or evaporation through a porous medium, J. Differential Equations 69 (1987), no. 3, 368-403.

[16] S. N. Kruzhkov, First-order quasilinear equations with several space variables, Math. USSR. Sb. 10 (1970), 217-242.

[17] F. Otto, $L^{1}$-contraction and uniqueness for quasilinear elliptic-parabolic equations, C. R. Acad. Sci. Paris Sér. I Math. 321 (1995), no. 8, 1005-1010.

[18] S. Ouaro, Entropy solutions of a stationary problem associated to a nonlinear parabolic strongly degenerate problem in one space dimension, An. Univ. Craiova Ser. Mat. Inform. 33 (2006), 108-131.

[19] _ Entropy solutions of nonlinear elliptic-parabolic-hyperbolic degenerate problems in one dimension, Int. J. Evol. Equ. 3 (2007), no. 1, 1-18. 